University of Nebraska - Lincoln

DigitalCommons@University of Nebraska - Lincoln

\title{
Environmental contaminants in Freshwater Fish and Their Risk to Piscivorous Wildlife Based on a National Monitoring Program
}

Jo Ellen Hinck

U.S. Geological Survey, jhinck@usgs.gov

Christopher J. Schmitt

U.S. Geological Survey, cjschmitt@usgs.gov

Kimberly A. Chojnacki

U.S. Geological Survey, kchojnacki@usgs.gov

Donald E. Tillitt

U.S. Geological Survey, dtillitt@usgs.gov

Follow this and additional works at: https://digitalcommons.unl.edu/usgsstaffpub

Part of the Earth Sciences Commons

Hinck, Jo Ellen; Schmitt, Christopher J.; Chojnacki, Kimberly A.; and Tillitt, Donald E., "Environmental contaminants in Freshwater Fish and Their Risk to Piscivorous Wildlife Based on a National Monitoring Program" (2009). USGS Staff -- Published Research. 17.

https://digitalcommons.unl.edu/usgsstaffpub/17

This Article is brought to you for free and open access by the US Geological Survey at DigitalCommons@University of Nebraska - Lincoln. It has been accepted for inclusion in USGS Staff -- Published Research by an authorized administrator of DigitalCommons@University of Nebraska - Lincoln. 


\title{
Environmental contaminants in freshwater fish and their risk to piscivorous wildlife based on a national monitoring program
}

\author{
Jo Ellen Hinck • Christopher J. Schmitt • \\ Kimberly A. Chojnacki • Donald E. Tillitt
}

Received: 18 November 2007 / Accepted: 10 April 2008/Published online: 2 July 2008

(C) Springer Science + Business Media B.V. 2008

\begin{abstract}
Organochlorine chemical residues and elemental concentrations were measured in piscivorous and benthivorous fish at 111 sites from large U.S. river basins. Potential contaminant sources such as urban and agricultural runoff, industrial discharges, mine drainage, and irrigation varied among the sampling sites. Our objectives were to provide summary statistics for chemical contaminants and to determine if contaminant concentrations in the fish were a risk to wildlife that forage at these sites. Concentrations of dieldrin, total DDT, total PCBs, toxaphene, TCDD$\mathrm{EQ}$, cadmium, chromium, mercury, lead, selenium, and zinc exceeded toxicity thresholds to protect fish and piscivorous wildlife in samples from at least one site; most exceedences were for total PCBs, mercury, and zinc. Chemical concentrations in fish from the Mississippi River Basin exceeded the greatest number of toxicity thresholds. Screening level wildlife risk analysis models were developed for bald eagle and mink using no adverse effect levels (NOAELs), which were derived from adult dietary exposure or tissue concentration studies and based primarily on reproductive endpoints. No effect hazard concentrations
\end{abstract}

J. E. Hinck $(\bowtie) \cdot$ C. J. Schmitt $\cdot$ K. A. Chojnacki •

D. E. Tillitt

U.S. Geological Survey (USGS),

Columbia Environmental Research Center (CERC),

4200 New Haven Rd.,

Columbia, MO 65201, USA

e-mail: jhinck@usgs.gov
(NEHC) were calculated by comparing the NOAEL to the food ingestion rate (dietary-based NOAEL) or biomagnification factor (tissue-based NOAEL) of each receptor. Piscivorous wildlife may be at risk from a contaminant if the measured concentration in fish exceeds the NEHC. Concentrations of most organochlorine residues and elemental contaminants represented no to low risk to bald eagle and mink at most sites. The risk associated with pentachloroanisole, aldrin, Dacthal, methoxychlor, mirex, and toxaphene was unknown because NOAELs for these contaminants were not available for bald eagle or mink. Risk differed among modeled species and sites. Our screening level analysis indicates that the greatest risk to piscivorous wildlife was from total DDT, total PCBs, TCDD-EQ, mercury, and selenium. Bald eagles were at greater risk to total DDT and total PCBs than mink, whereas risks of TCDD-EQ, mercury, and selenium were greater to mink than bald eagle.

Keywords Biomonitoring $\cdot$ Ecological risk assessment $\cdot$ Toxicity $\cdot$ Dietary exposure · Biomagnification

\section{Introduction}

The bioaccumulation of contaminants by fish represents a potentially significant risk to piscivorous wildlife. Persistent, accumulative substances such as DDT (as DDE), 
polychlorinated biphenyls (PCBs), mercury, and selenium can accumulate in fish and pose a threat to piscivorous wildlife. Fish often comprise a substantial dietary portion of species such as mink (Mustela vison), river otter (Lutra canadensis), osprey (Pandion haliaetus), and bald eagle (Haliaeetus leucocephalus), and therefore represent a significant route of contaminant exposure. In addition, wildlife typically consume most or all of the fish, including internal organs, in which concentrations of many contaminants are generally greater than those in the muscle tissue (fillet) typically used to assess human health risks.

The Large River Monitoring Network (LRMN) of the Biomonitoring of Environmental Status and Trends (BEST) Project was initiated to document spatial trends in accumulative chemical contaminants, health indicators, and reproductive biomarkers. From 1995 through 2004, the BEST-LRMN Project measured concentrations of organochlorine pesticides, PCBs, and elemental contaminants in whole-body composite samples of piscivorous and benthivorous fish from nine U.S. river basins (Schmitt 2002; 2004; Schmitt et al. 2005; Hinck et al. 2006a, b; 2007, 2008). Hinck et al. (2006b) determined that avian and mammalian piscivorous wildlife were at risk to mercury, but not organochlorine pesticides or PCBs, in northern pike (Esox lucius), longnose sucker (Catostomus catostomus), and burbot (Lota lota) from the Yukon River Basin. However, the risk of chemical contaminants to piscivorous wildlife that live and forage at BEST-LRMN sites in the conterminous United States has not been evaluated. Therefore, this paper reports a wildlife risk analysis that was conducted to determine the risk of these contaminants to avian (bald eagle) and mammalian (mink) receptors that consume the large adult fish such as those collected by the BEST-LRMN Project and have a broad geographic range. Unlike many ecological risk assessments that estimate fish contaminant concentrations from sediment or soil contaminant concentrations, contaminant concentrations in whole-body fish samples from the BEST-LRMN Project were used in the analysis to give a more accurate estimate of risk to piscivorous wildlife.

The BEST-LRMN Project was preceded by the National Contaminant Biomonitoring Program (NCBP). The NCBP measured organochlorine residues and elemental contaminants in whole-body fish composite samples from 1976 through 1986 at 107 locations across the United States (Schmitt et al. 1999). The NCBP demonstrated that concentrations of organochlorine pesticides, PCBs, and certain elemental contaminants such as mercury were generally decreasing in fish and provided descriptive statistics for concentration distributions in fish from across the United States. The geometric means and 85th percentile concentrations from the NCBP have been previously used as benchmarks for national trends of contaminant concentrations in whole-body fish (Schmitt et al. 1999). Some of the BEST-LRMN sites were co-located at historical NCBP sites, and trend analysis for contaminant concentrations were included in individual basin reports (Schmitt 2002; 2004; Schmitt et al. 2005; Hinck et al. 2006a; 2007).

This paper provides an example of how contaminant concentrations in fish from a routine monitoring program can be used to screen for risk of contaminant exposure to piscivorous wildlife. This approach follows the procedures developed by the U.S. Environmental Protection Agency (USEPA) to conduct screening-level ecological risk assessments (USEPA 1998). Although these assessments are usually applied locally to assess specific sites, they have been used in regional assessments. For example, Yeardley et al. (1998) examined the risk of mercury to wildlife in northeastern U.S. lakes, and Hinck et al. (2006b) determined the risk of contaminant exposure to avian and mammalian receptors in the Yukon River Basin. Our objectives were (1) to provide summary statistics for organochlorine chemical residues and elemental contaminants measured by the BESTLRMN Project and (2) to screen for contaminants that represent a risk to bald eagle and mink and identify the areas where the risk to these receptors was greatest. The wildlife risk analysis data from Hinck et al. (2006b) were updated in this paper to provide results for all BEST-LRMN samples. These results were then compared to site specific studies to determine if piscivorous wildlife had previously been identified as at risk to contaminant exposure.

\section{Materials and methods}

Detailed sampling, field, and laboratory procedures have been previously described (Schmitt 2002; 2004; Schmitt et al. 2005; Hinck et al. 2006a, b; 2007; 2008). A summary is presented here. 
Sampling and field procedures

Fish were collected from 111 sites in nine river basins (Table 1; Fig. 1). Sites were located in the Apalachicola River Basin $(n=3)$, Colorado River Basin $(n=$ 14), Columbia River Basin $(n=16)$, Mobile River Basin $(n=4)$, Mississippi River Basin $(n=48)$, Pee Dee River Basin $(n=3)$, Rio Grande Basin $(n=10)$, Savannah River Basin $(n=3)$, and Yukon River Basin $(n=10$; Table 1). Of these, 51 were NCBP sites that had been sampled historically (Schmitt et al. 1999). Each site was sampled once. Sampling sites were located on the mainstem and large tributaries of the river basins with known access points without regard to contaminant sources; however, the sites did represent a range of contaminant sources (e.g., chemical manufacturing, agricultural, and urban areas). Most fish were captured by electrofishing; hook-and-line, gill nets, and fyke nets were used only in the Yukon River Basin. Piscivorous species included black bass (Micropterus spp.), white bass (Morone spp.), catfish (Ictaluridae), northern pike, northern pikeminnow (Ptychocheilus oregonensis), burbot, trout (Salmonidae), pikeperch (Sander spp.), and goldeye (Hiodon alosoides). Benthivorous species included common carp (Cyprinus carpio) and sucker (Catistomidae; Table 2).

\section{Laboratory analyses}

Fish were shipped to the laboratory frozen on dry ice and stored at $-20^{\circ} \mathrm{C}$ until prepared for analysis. Individual whole fish were partly thawed, cut into pieces, and ground to a fine texture. The ground subsamples were then grouped to create a single homogenous composite sample for each site, species, and gender combination. A total of 409 composite samples were included in the chemical analysis.

One sub-sample $(10 \mathrm{~g})$ of each composite sample was solvent-extracted and analyzed gravimetrically for lipid content and by high-resolution capillary gas chromatography with electron capture detection for organochlorine pesticide residues and total PCBs (sum of individual congeners) after size exclusion and adsorption column cleanup. Pentachlorobenzene, pentachloroanisole, aldrin, Dacthal, heptachlor, endosulfan I, endosulfan II, endosulfan sulfate, and methoxychlor were not measured in Columbia, Rio Grande, and Mississippi River Basin samples. Quality assurance (QA) measures for the organochlorine pesticide and PCB analyses included the analysis of blanks, triplicate analyses, and matrix spikes. The detection limit for each compound was calculated by adding the average procedural blank concentration to three times the procedural blank standard deviation (Keith 1991) and varied among study years with detection limits changing by two orders of magnitude for some organochlorine residues (Table 3 ). Concentrations were converted from ng/g wet weight (ww) to $\mu \mathrm{g} / \mathrm{g}$ ww for statistical and wildlife risk analyses.

A second sub-sample of each fish composite sample $(100 \mathrm{~g})$ was freeze-dried for elemental analysis. Percent moisture was determined as weight lost during lyophilization. For samples from the Mississippi, Rio Grande, and Columbia River Basins, one portion of the dried material was acid-digested and analyzed by atomic absorption spectroscopy and inductively coupled plasma emission spectroscopy. For all other samples, three sub-samples were analyzed for elemental contaminants. One portion of the dried material was digested in nitric acid and analyzed by inductively coupled plasma mass spectroscopy for cadmium, copper, chromium, nickel, lead, and zinc. A second portion was dry-ashed (magnesium nitrate-nitric acid-HCl) and analyzed by hydride generation atomic absorption spectroscopy for arsenic and selenium. A third portion was analyzed directly for total mercury using thermal combustion, amalgamation, and atomic absorption spectroscopy. QA measures for the elemental analyses included the analysis of reagent blanks, duplicate samples, certified reference materials, and fortified samples. Dry-weight (dw) detection limits were determined individually for each element in each sample. Elemental concentrations (including detection limits) were converted from $\mu \mathrm{g} / \mathrm{g} d \mathrm{~d}$ to $\mu \mathrm{g} / \mathrm{g}$ ww for statistical analysis and reporting using the moisture content of each sample (Table 4).

A third sub-sample $(10 \mathrm{~g})$ was solvent-extracted and subjected to reactive cleanup for use in the H4IIE bioassay (Tillitt et al. 1991; Whyte et al. 2004). Concentrations of 2,3,7,8-tetrachlorodibenzo- $p$-dioxin equivalents (TCDD-EQ; pg/g ww) were determined by slope ratio assay as modified by Ankley et al. (1991). QA measures for the H4IIE bioassay included analysis of duplicate samples and reference materials. Concentrations were converted to $\mu \mathrm{g} / \mathrm{g}$ ww for the wildlife risk analysis. 
Table 1 Freshwater fish collection sites of the BEST-LRMN Project

\begin{tabular}{|c|c|c|c|}
\hline Basin site no. & River & Nearest city or feature & Latitude longitude \\
\hline \multicolumn{4}{|c|}{ Apalachicola River Basin } \\
\hline 330 & Chattahoochee & Omaha, GA & $32^{\circ} 13^{\prime} 19.80^{\prime \prime} \mathrm{N}, 84^{\circ} 55^{\prime} 35.10^{\prime \prime} \mathrm{W}$ \\
\hline 331 & Flint & Albany, GA & $31^{\circ} 34^{\prime} 34.86^{\prime \prime} \mathrm{N}, 84^{\circ} 08^{\prime} 49.80^{\prime \prime} \mathrm{W}$ \\
\hline 332 & Apalachicola & Blountstown, FL & $30^{\circ} 25^{\prime} 58.20^{\prime \prime} \mathrm{N}, 85^{\circ} 01^{\prime} 17.10^{\prime \prime} \mathrm{W}$ \\
\hline \multicolumn{4}{|c|}{ Colorado River Basin } \\
\hline 311 & Yampa & Lay, CO & $40^{\circ} 25^{\prime} 38.00^{\prime \prime} \mathrm{N}, 107^{\circ} 51^{\prime} 24.00^{\prime \prime} \mathrm{W}$ \\
\hline 312 & Green & Ouray NWR, UT & $40^{\circ} 08^{\prime} 31.00^{\prime \prime} \mathrm{N}, 109^{\circ} 39^{\prime} 17.00^{\prime \prime} \mathrm{W}$ \\
\hline 313 & Green & San Rafael, UT & $38^{\circ} 45^{\prime} 56.00^{\prime \prime} \mathrm{N}, 110^{\circ} 05^{\prime} 16.00^{\prime \prime} \mathrm{W}$ \\
\hline 314 & Gunnison & Delta, CO & $38^{\circ} 45^{\prime} 59.58^{\prime \prime} \mathrm{N}, 108^{\circ} 02^{\prime} 30.30^{\prime \prime} \mathrm{W}$ \\
\hline 315 & Colorado & Loma, CO & $39^{\circ} 09^{\prime} 39.00^{\prime \prime} \mathrm{N}, 108^{\circ} 48^{\prime} 28.56^{\prime \prime} \mathrm{W}$ \\
\hline 316 & Colorado & Gold Bar Canyon, UT & $38^{\circ} 34^{\prime} 02.00^{\prime \prime} \mathrm{N}, 109^{\circ} 38^{\prime} 51.00^{\prime \prime} \mathrm{W}$ \\
\hline 317 & San Juan & Hogback Diversion, NM & $36^{\circ} 44^{\prime} 41.00^{\prime \prime} \mathrm{N}, 108^{\circ} 41^{\prime} 28.00^{\prime \prime} \mathrm{W}$ \\
\hline 319 & Colorado & South Cove, AZ & $36^{\circ} 05^{\prime} 23.70^{\prime \prime} \mathrm{N}, 114^{\circ} 06^{\prime} 12.30^{\prime \prime} \mathrm{W}$ \\
\hline 320 & Colorado & Willow Beach, AZ & $35^{\circ} 52^{\prime} 33.12^{\prime \prime} \mathrm{N}, 114^{\circ} 39^{\prime} 53.10^{\prime \prime} \mathrm{W}$ \\
\hline 321 & Colorado & Needles, CA & $34^{\circ} 43^{\prime} 44.64^{\prime \prime} \mathrm{N}, 114^{\circ} 20^{\prime} 12.96^{\prime \prime} \mathrm{W}$ \\
\hline 322 & Colorado & Imperial Dam, AZ & $32^{\circ} 54^{\prime} 05.94^{\prime \prime} \mathrm{N}, 114^{\circ} 28^{\prime} 09.48^{\prime \prime} \mathrm{W}$ \\
\hline 323 & Gila & Hayden, AZ & $33^{\circ} 01^{\prime} 22.14^{\prime \prime} \mathrm{N}, 110^{\circ} 44^{\prime} 16.32^{\prime \prime} \mathrm{W}$ \\
\hline 324 & Gila & Phoenix, AZ & $33^{\circ} 22^{\prime} 33.42^{\prime \prime} \mathrm{N}, 112^{\circ} 18^{\prime} 19.20^{\prime \prime} \mathrm{W}$ \\
\hline 325 & Gila & Arlington, AZ & $33^{\circ} 19^{\prime} 06.92^{\prime \prime} \mathrm{N}, 112^{\circ} 40^{\prime} 26.46^{\prime \prime} \mathrm{W}$ \\
\hline \multicolumn{4}{|c|}{ Columbia River Basin } \\
\hline 41 & Snake & Hagerman, ID & $42^{\circ} 47^{\prime} 36.21^{\prime \prime} \mathrm{N}, 114^{\circ} 56^{\prime} 18.10^{\prime \prime} \mathrm{W}$ \\
\hline 42 & Salmon & Riggins, ID & $45^{\circ} 35^{\prime} 43.42^{\prime \prime} \mathrm{N}, 116^{\circ} 16^{\prime} 55.00^{\prime \prime} \mathrm{W}$ \\
\hline 43 & Snake & Lewiston, ID & $46^{\circ} 24^{\prime} 54.28^{\prime \prime} \mathrm{N}, 117^{\circ} 02^{\prime} 03.49^{\prime \prime} \mathrm{W}$ \\
\hline 44 & Yakima & Granger, WA & $46^{\circ} 20^{\prime} 49.31^{\prime \prime} \mathrm{N}, 120^{\circ} 12^{\prime} 27.03^{\prime \prime} \mathrm{W}$ \\
\hline 45 & Willamette & Oregon City, OR & $45^{\circ} 19^{\prime} 03.47^{\prime \prime} \mathrm{N}, 122^{\circ} 39^{\prime} 57.50^{\prime \prime} \mathrm{W}$ \\
\hline 46 & Columbia & Cascade Locks, OR & $45^{\circ} 41^{\prime} 23.11^{\prime \prime} \mathrm{N}, 121^{\circ} 51^{\prime} 00.41^{\prime \prime} \mathrm{W}$ \\
\hline 96 & Snake & Ice Harbor Dam, WA & $46^{\circ} 41^{\prime} 51.68^{\prime \prime} \mathrm{N}, 118^{\circ} 53^{\prime} 07.88^{\prime \prime} \mathrm{W}$ \\
\hline 97 & Columbia & Pasco, WA & $46^{\circ} 31^{\prime} 49.22^{\prime \prime} \mathrm{N}, 119^{\circ} 16^{\prime} 42.07^{\prime \prime} \mathrm{W}$ \\
\hline 98 & Columbia & Grand Coulee, WA & $47^{\circ} 57^{\prime} 44.85^{\prime \prime} \mathrm{N}, 118^{\circ} 58^{\prime} 53.84^{\prime \prime} \mathrm{W}$ \\
\hline 117 & Flathead & Creston, MT & $48^{\circ} 09^{\prime} 01.09^{\prime \prime} \mathrm{N}, 114^{\circ} 11^{\prime} 29.71^{\prime \prime} \mathrm{W}$ \\
\hline 501 & Columbia & Beaver Army Terminal, OR & $46^{\circ} 10^{\prime} 57.86^{\prime \prime} \mathrm{N}, 123^{\circ} 04^{\prime} 13.87^{\prime \prime} \mathrm{W}$ \\
\hline 502 & Columbia & Warrendale, OR & $45^{\circ} 38^{\prime} 00.82^{\prime \prime} \mathrm{N}, 121^{\circ} 58^{\prime} 42.57^{\prime \prime} \mathrm{W}$ \\
\hline 503 & Columbia & Vernita Bridge, WA & $46^{\circ} 37^{\prime} 28.40^{\prime \prime} \mathrm{N}, 119^{\circ} 51^{\prime} 31.45^{\prime \prime} \mathrm{W}$ \\
\hline 504 & Columbia & Northport, WA & $48^{\circ} 58^{\prime} 21.70^{\prime \prime} \mathrm{N}, 117^{\circ} 38^{\prime} 48.92^{\prime \prime} \mathrm{W}$ \\
\hline 505 & Willamette & Portland, OR & $45^{\circ} 33^{\prime} 04.51^{\prime \prime} \mathrm{N}, 122^{\circ} 41^{\prime} 43.74^{\prime \prime} \mathrm{W}$ \\
\hline 506 & Columbia & Vancouver, WA & $45^{\circ} 35^{\prime} 44.21^{\prime \prime} \mathrm{N}, 122^{\circ} 32^{\prime} 13.61^{\prime \prime} \mathrm{W}$ \\
\hline \multicolumn{4}{|c|}{ Mobile River Basin } \\
\hline 326 & Tombigbee & Lavaca, AL & $32^{\circ} 15^{\prime} 53.60^{\prime \prime} \mathrm{N}, 88^{\circ} 00^{\prime} 44.21^{\prime \prime} \mathrm{W}$ \\
\hline 327 & Coosa & Childersburg, AL & $33^{\circ} 19^{\prime} 57.76^{\prime \prime} \mathrm{N}, 86^{\circ} 21^{\prime} 55.87^{\prime \prime} \mathrm{W}$ \\
\hline 328 & Alabama & Eureka Landing, AL & $31^{\circ} 23^{\prime} 14.06^{\prime \prime} \mathrm{N}, 87^{\circ} 42^{\prime} 42.19^{\prime \prime} \mathrm{W}$ \\
\hline 329 & Mobile & Bucks, AL & $31^{\circ} 03^{\prime} 15.85^{\prime \prime} \mathrm{N}, 87^{\circ} 59^{\prime} 48.07^{\prime \prime} \mathrm{W}$ \\
\hline \multicolumn{4}{|c|}{ Mississippi River Basin } \\
\hline 15 & Mississippi & Luling, LA & $29^{\circ} 59^{\prime} 53.2^{\prime \prime} \mathrm{N}, 90^{\circ} 25^{\prime} 31.1^{\prime \prime} \mathrm{W}$ \\
\hline 23 & Kanawha & Winfield, WV & $38^{\circ} 29^{\prime} 06.0^{\prime \prime} \mathrm{N}, 81^{\circ} 48^{\prime} 57.6^{\prime \prime} \mathrm{W}$ \\
\hline 24 & Ohio & Marietta, $\mathrm{OH}$ & $39^{\circ} 24^{\prime} 36.8^{\prime \prime} \mathrm{N}, 81^{\circ} 26^{\prime} 26.3^{\prime \prime} \mathrm{W}$ \\
\hline 25 & Cumberland & Clarksville, $\mathrm{OH}$ & $36^{\circ} 32^{\prime} 28.6^{\prime \prime} \mathrm{N}, 87^{\circ} 22^{\prime} 04.7^{\prime \prime} \mathrm{W}$ \\
\hline 26 & Illinois & Beardstown, IL & $40^{\circ} 07^{\prime} 50.6^{\prime \prime} \mathrm{N}, 90^{\circ} 20^{\prime} 45.6^{\prime \prime} \mathrm{W}$ \\
\hline 27 & Mississippi & Guttenburg, IA & $42^{\circ} 43^{\prime} 37.2^{\prime \prime} \mathrm{N}, 91^{\circ} 01^{\prime} 30.0^{\prime \prime} \mathrm{W}$ \\
\hline 28 & Arkansas & Pine Bluff, AR & $34^{\circ} 16^{\prime} 27.0^{\prime \prime} \mathrm{N}, 94^{\circ} 57^{\prime} 12.0^{\prime \prime} \mathrm{W}$ \\
\hline
\end{tabular}


Table 1 (continued)

\begin{tabular}{|c|c|c|c|}
\hline Basin site no. & River & Nearest city or feature & Latitude longitude \\
\hline 29 & Arkansas & Keystone Res., OK & $36^{\circ} 07^{\prime} 54.0^{\prime \prime} \mathrm{N}, 96^{\circ} 20^{\prime} 47.0^{\prime \prime} \mathrm{W}$ \\
\hline 30 & White & Devall's Bluff, AR & $34^{\circ} 47^{\prime} 01.0^{\prime \prime} \mathrm{N}, 91^{\circ} 26^{\prime} 28.0^{\prime \prime} \mathrm{W}$ \\
\hline 31 & Missouri & Nebraska City, NE & $40^{\circ} 40^{\prime} 15.9^{\prime \prime} \mathrm{N}, 95^{\circ} 49^{\prime} 44.6^{\prime \prime} \mathrm{W}$ \\
\hline 32 & Missouri & Garrison Dam, ND & $47^{\circ} 28^{\prime} 27.3^{\prime \prime} \mathrm{N}, 101^{\circ} 26^{\prime} 15.5^{\prime \prime} \mathrm{W}$ \\
\hline 67 & Allegheny & Natrona, PA & $40^{\circ} 39^{\prime} 54.0^{\prime \prime} \mathrm{N}, 79^{\circ} 41^{\prime} 24.0^{\prime \prime} \mathrm{W}$ \\
\hline 68 & Wabash & New Harmony, IN & $38^{\circ} 11^{\prime} 58.4^{\prime \prime} \mathrm{N}, 87^{\circ} 58^{\prime} 36.0^{\prime \prime} \mathrm{W}$ \\
\hline 70 & Ohio & Metropolis, IL & $37^{\circ} 07^{\prime} 40.8^{\prime \prime} \mathrm{N}, 88^{\circ} 39^{\prime} 25.2^{\prime \prime} \mathrm{W}$ \\
\hline 71 & Tennessee & Savannah, TN & $35^{\circ} 12^{\prime} 52.0^{\prime \prime} \mathrm{N}, 88^{\circ} 18^{\prime} 36.0^{\prime \prime} \mathrm{W}$ \\
\hline 72 & Wisconsin & Woodman, WI & $43^{\circ} 05^{\prime} 42.0^{\prime \prime} \mathrm{N}, 90^{\circ} 48^{\prime} 57.6^{\prime \prime} \mathrm{W}$ \\
\hline 73 & Des Moines & Keosauqua, IA & $40^{\circ} 44^{\prime} 52.8^{\prime \prime} \mathrm{N}, 91^{\circ} 59^{\prime} 38.4^{\prime \prime} \mathrm{W}$ \\
\hline 74 & Mississippi & Little Falls, MN & $45^{\circ} 58^{\prime} 48.0^{\prime \prime} \mathrm{N}, 94^{\circ} 22^{\prime} 00.0^{\prime \prime} \mathrm{W}$ \\
\hline 75 & Mississippi & Cape Girardeau, MO & $37^{\circ} 18^{\prime} 36.0^{\prime \prime} \mathrm{N}, 89^{\circ} 31^{\prime} 01.2^{\prime \prime} \mathrm{W}$ \\
\hline 76 & Mississippi & Memphis, TN & $38^{\circ} 08^{\prime} 30.3^{\prime \prime} \mathrm{N}, 90^{\circ} 03^{\prime} 36.6^{\prime \prime} \mathrm{W}$ \\
\hline 77 & Arkansas & John Martin Res., CO & $38^{\circ} 03^{\prime} 55.0^{\prime \prime} \mathrm{N}, 102^{\circ} 56^{\prime} 02.0^{\prime \prime} \mathrm{W}$ \\
\hline 78 & Verdigris & Oolagah, OK & $36^{\circ} 31^{\prime} 16.0^{\prime \prime} \mathrm{N}, 95^{\circ} 33^{\prime} 37.0^{\prime \prime} \mathrm{W}$ \\
\hline 79 & Canadian & Eufaula, OK & $35^{\circ} 16^{\prime} 43.0^{\prime \prime} \mathrm{N}, 95^{\circ} 34^{\prime} 39.0^{\prime \prime} \mathrm{W}$ \\
\hline 80 & Yazoo & Redwood, MS & $32^{\circ} 24^{\prime} 36.0^{\prime \prime} \mathrm{N}, 90^{\circ} 55^{\prime} 27.0^{\prime \prime} \mathrm{W}$ \\
\hline 81 & Red & Alexandria, LA & $31^{\circ} 20^{\prime} 48.0^{\prime \prime} \mathrm{N}, 92^{\circ} 27^{\prime} 37.0^{\prime \prime} \mathrm{W}$ \\
\hline 82 & Red & Lake Texoma, TX/OK & $33^{\circ} 52^{\prime} 08.0^{\prime \prime} \mathrm{N}, 96^{\circ} 47^{\prime} 04.0^{\prime \prime} \mathrm{W}$ \\
\hline 83 & Missouri & Hermann, MO & $38^{\circ} 42^{\prime} 24.1^{\prime \prime} \mathrm{N}, 91^{\circ} 26^{\prime} 17.5^{\prime \prime} \mathrm{W}$ \\
\hline 84 & Big Horn & Hardin, MT & $45^{\circ} 52^{\prime} 12.2^{\prime \prime} \mathrm{N}, 107^{\circ} 34^{\prime} 34.0^{\prime \prime} \mathrm{W}$ \\
\hline 85 & Yellowstone & Sidney, NE & $47^{\circ} 34^{\prime} 46.8^{\prime \prime} \mathrm{N}, 104^{\circ} 13^{\prime} 10.7^{\prime \prime} \mathrm{W}$ \\
\hline 86 & James & Olivet, SD & $43^{\circ} 13^{\prime} 45.0^{\prime \prime} \mathrm{N}, 97^{\circ} 41^{\prime} 05.0^{\prime \prime} \mathrm{W}$ \\
\hline 89 & Platte & Louisville, NE & $40^{\circ} 59^{\prime} 33.1^{\prime \prime} \mathrm{N}, 96^{\circ} 12^{\prime} 30.9^{\prime \prime} \mathrm{W}$ \\
\hline 90 & Kansas & Bonner Springs, KS & $39^{\circ} 02^{\prime} 47.0^{\prime \prime} \mathrm{N}, 94^{\circ} 47^{\prime} 05.0^{\prime \prime} \mathrm{W}$ \\
\hline 111 & Mississippi & Lake City, MN & $44^{\circ} 22^{\prime} 49.8^{\prime \prime} \mathrm{N}, 92^{\circ} 07^{\prime} 33.0^{\prime \prime} \mathrm{W}$ \\
\hline 112 & Mississippi & Dubuque, IA & $42^{\circ} 26^{\prime} 27.6^{\prime \prime} \mathrm{N}, 90^{\circ} 35^{\prime} 06.0^{\prime} \mathrm{W}$ \\
\hline 201 & Big Sunflower & Anguilla, MS & $32^{\circ} 58^{\prime} 18.0^{\prime \prime} \mathrm{N}, 90^{\circ} 46^{\prime} 40.0^{\prime \prime} \mathrm{W}$ \\
\hline 202 & Bogue Phalia & Leland, MS & $33^{\circ} 24^{\prime} 22.0^{\prime \prime} \mathrm{N}, 90^{\circ} 50^{\prime} 26.0^{\prime \prime} \mathrm{W}$ \\
\hline 203 & Steele Bayou & Rolling Fork, MS & $32^{\circ} 54^{\prime} 71.0^{\prime \prime} \mathrm{N}, 90^{\circ} 57^{\prime} 10.0^{\prime \prime} \mathrm{W}$ \\
\hline 204 & Tensas & Tendal, LA & $32^{\circ} 25^{\prime} 56.0^{\prime \prime} \mathrm{N}, 91^{\circ} 21^{\prime} 57.0^{\prime \prime} \mathrm{W}$ \\
\hline 205 & S. Skunk & Oskaloosa, IA & $41^{\circ} 21^{\prime} 19.0^{\prime \prime} \mathrm{N}, 92^{\circ} 39^{\prime} 31.0^{\prime \prime} \mathrm{W}$ \\
\hline 206 & Iowa & Morengo, IA & $41^{\circ} 50^{\prime} 23.0^{\prime \prime} \mathrm{N}, 92^{\circ} 11^{\prime} 54.0^{\prime \prime} \mathrm{W}$ \\
\hline 207 & Cache & Cotton Plant, AR & $35^{\circ} 02^{\prime} 32.0^{\prime \prime} \mathrm{N}, 91^{\circ} 19^{\prime} 12.0^{\prime \prime} \mathrm{W}$ \\
\hline 208 & Cache & Egypt, AR & $35^{\circ} 51^{\prime} 23.0^{\prime \prime} \mathrm{N}, 90^{\circ} 56^{\prime} 15.0^{\prime \prime} \mathrm{W}$ \\
\hline 209 & S. Fork Iowa & New Providence, IA & $42^{\circ} 19^{\prime} 26.0^{\prime \prime} \mathrm{N}, 93^{\circ} 10^{\prime} 10.0^{\prime \prime} \mathrm{W}$ \\
\hline 210 & Iowa & Rowan, IA & $42^{\circ} 45^{\prime} 36.0^{\prime \prime} \mathrm{N}, 93^{\circ} 37^{\prime} 23.0^{\prime \prime} \mathrm{W}$ \\
\hline 211 & Cedar & St. Charles City, IA & $43^{\circ} 03^{\prime} 45.0^{\prime \prime} \mathrm{N}, 92^{\circ} 40^{\prime} 23.0^{\prime \prime} \mathrm{W}$ \\
\hline 212 & Little R. Ditch & Moorehouse, MO & $36^{\circ} 50^{\prime} 03.0^{\prime \prime} \mathrm{N}, 89^{\circ} 43^{\prime} 48.0^{\prime \prime} \mathrm{W}$ \\
\hline 213 & Wolf & LaGrange, TN & $35^{\circ} 01^{\prime} 57.0^{\prime \prime} \mathrm{N}, 89^{\circ} 14^{\prime} 48.0^{\prime \prime} \mathrm{W}$ \\
\hline $400^{\mathrm{a}}$ & Leetown Res. & Kearneysville, WV & $39^{\circ} 21^{\prime} 2.15^{\prime \prime} \mathrm{N}, 7^{\circ} 55^{\prime} 32.69^{\prime \prime} \mathrm{W}$ \\
\hline \multicolumn{4}{|c|}{ Pee Dee River Basin } \\
\hline 336 & Pee Dee & Rockingham, NC & $34^{\circ} 53^{\prime} 22.14^{\prime \prime} \mathrm{N}, 79^{\circ} 51^{\prime} 24.89^{\prime \prime} \mathrm{W}$ \\
\hline 337 & Pee Dee & Pee Dee, SC & $34^{\circ} 21^{\prime} 23.22^{\prime \prime} \mathrm{N}, 79^{\circ} 41^{\prime} 35.19^{\prime \prime} \mathrm{W}$ \\
\hline 338 & Pee Dee & Bucksport, SC & $33^{\circ} 42^{\prime} 18.09^{\prime \prime} \mathrm{N}, 79^{\circ} 11^{\prime} 24.00^{\prime \prime} \mathrm{W}$ \\
\hline \multicolumn{4}{|c|}{ Rio Grande Basin } \\
\hline 16 & Rio Grande & Mission, TX & $26^{\circ} 09^{\prime} 28.74^{\prime \prime} \mathrm{N}, 98^{\circ} 20^{\prime} 02.82^{\prime \prime} \mathrm{W}$ \\
\hline 63 & Rio Grande & Elephant Butte Res., NM & $33^{\circ} 12^{\prime} 48.55^{\prime \prime} \mathrm{N}, 107^{\circ} 13^{\prime} 27.26^{\prime \prime} \mathrm{W}$ \\
\hline 64 & Rio Grande & Alamosa, CO & $37^{\circ} 25^{\prime} 06.42^{\prime \prime} \mathrm{N}, 37^{\circ} 23^{\prime} 38.57^{\prime \prime} \mathrm{W}$ \\
\hline 65 & Pecos & Red Bluff Lake, TX & $32^{\circ} 00^{\prime} 00.00^{\prime \prime} \mathrm{N}, 104^{\circ} 58^{\prime} 30.00^{\prime \prime} \mathrm{W}$ \\
\hline
\end{tabular}


Table 1 (continued)

\begin{tabular}{|c|c|c|c|}
\hline Basin site no. & River & Nearest city or feature & Latitude longitude \\
\hline 511 & Arroyo Colorado & Harlingen, TX & $26^{\circ} 11^{\prime} 44.28^{\prime \prime} \mathrm{N}, 97^{\circ} 36^{\prime} 20.52^{\prime \prime} \mathrm{W}$ \\
\hline 512 & Rio Grande & Brownsville, TX & $25^{\circ} 52^{\prime} 12.96^{\prime \prime} \mathrm{N}, 97^{\circ} 27^{\prime} 06.30^{\prime \prime} \mathrm{W}$ \\
\hline 513 & Rio Grande & Below Falcon Dam, TX & $20^{\circ} 08^{\prime} 06.66^{\prime \prime} \mathrm{N}, 99^{\circ} 08^{\prime} 06.42^{\prime \prime} \mathrm{W}$ \\
\hline 514 & Rio Grande & Below Amistad Res., TX & $29^{\circ} 26^{\prime} 49.06^{\prime \prime} \mathrm{N}, 101^{\circ} 03^{\prime} 10.58^{\prime \prime} \mathrm{W}$ \\
\hline 515 & Rio Grande & Foster Ranch, Langtry, TX & $29^{\circ} 46^{\prime} 40.91^{\prime \prime} \mathrm{N}, 101^{\circ} 45^{\prime} 13.22^{\prime \prime} \mathrm{W}$ \\
\hline 516 & Rio Grande & El Paso, TX & $31^{\circ} 47^{\prime} 55.00^{\prime \prime} \mathrm{N}, 31^{\circ} 48^{\prime} 15.00^{\prime \prime} \mathrm{W}$ \\
\hline \multicolumn{4}{|c|}{ Savannah River Basin } \\
\hline 333 & Savannah & Augusta, GA & $33^{\circ} 22^{\prime} 00.18^{\prime \prime} \mathrm{N}, 81^{\circ} 56^{\prime} 46.44^{\prime \prime} \mathrm{W}$ \\
\hline 334 & Savannah & Sylvania, GA & $33^{\circ} 01^{\prime} 16.86^{\prime \prime} \mathrm{N}, 81^{\circ} 31^{\prime} 04.50^{\prime \prime} \mathrm{W}$ \\
\hline 335 & Savannah & Port Wentworth, GA & $32^{\circ} 13^{\prime} 26.34^{\prime \prime} \mathrm{N}, 81^{\circ} 08^{\prime} 47.04^{\prime \prime} \mathrm{W}$ \\
\hline \multicolumn{4}{|c|}{ Yukon River Basin } \\
\hline 301 & Yukon & Eagle, AK & $65^{\circ} 22^{\prime} 28.56^{\prime \prime} \mathrm{N}, 142^{\circ} 30^{\prime} 20.88^{\prime \prime} \mathrm{W}$ \\
\hline 302 & Porcupine & Fish Hook Bend, AK & $67^{\circ} 12^{\prime} 50.94^{\prime \prime} \mathrm{N}, 142^{\circ} 09^{\prime} 58.56^{\prime \prime} \mathrm{W}$ \\
\hline 303 & Porcupine & Fort Yukon, AK & $66^{\circ} 35^{\prime} 21.06^{\prime \prime} \mathrm{N}, 145^{\circ} 19^{\prime} 59.40^{\prime \prime} \mathrm{W}$ \\
\hline 304 & Ray & Near the Bridge, AK & $65^{\circ} 53^{\prime} 01.26^{\prime \prime} \mathrm{N}, 149^{\circ} 48^{\prime} 00.72^{\prime \prime} \mathrm{W}$ \\
\hline 305 & Tanana & Fairbanks, AK & $64^{\circ} 45^{\prime} 02.52^{\prime \prime} \mathrm{N}, 148^{\circ} 03^{\prime} 15.84^{\prime \prime} \mathrm{W}$ \\
\hline 306 & Tolovana & Nenana, AK & $64^{\circ} 56^{\prime} 56.40^{\prime \prime} \mathrm{N}, 149^{\circ} 44^{\prime} 13.44^{\prime \prime} \mathrm{W}$ \\
\hline 307 & Yukon & Tanana, AK & $65^{\circ} 08^{\prime} 09.72^{\prime \prime} \mathrm{N}, 152^{\circ} 24^{\prime} 54.72^{\prime \prime} \mathrm{W}$ \\
\hline 308 & Yukon & Galena, AK & $64^{\circ} 41^{\prime} 56.22^{\prime \prime} \mathrm{N}, 156^{\circ} 58^{\prime} 36.30^{\prime \prime} \mathrm{W}$ \\
\hline 309 & Innoko & Innoko NWR, AK & $63^{\circ} 38^{\prime} 33.66^{\prime \prime} \mathrm{N}, 158^{\circ} 00^{\prime} 43.56^{\prime \prime} \mathrm{W}$ \\
\hline 310 & Yukon & Kotlik, AK & $63^{\circ} 02^{\prime} 12.18^{\prime \prime} \mathrm{N}, 163^{\circ} 34^{\prime} 22.56^{\prime \prime} \mathrm{W}$ \\
\hline
\end{tabular}

${ }^{\mathrm{a}}$ Reference site in original study (Schmitt 2002)

Summary statistics including the number of detected concentrations, unweighted geometric means, median, and maximum concentrations were computed to examine distributions of each chemical contaminant for the entire dataset. The 85 th percentile also was computed to compare to historical NCBP concentrations (Schmitt et al. 1999). Organochlorine residues and elemental contaminant concentrations were compared to toxicity thresholds based on wholebody fish concentrations and wildlife toxicity values from the scientific literature. Criteria for inclusion of a toxicity threshold in our evaluation were that the threshold was based on a whole-body fish concentration and associated with reproductive performance, growth, or survival. Wildlife toxicity values were used when whole-body toxicity threshold were not available. Censored values (i.e., concentrations less than the detection limit) were replaced by one half the detection limit in all figures.

Screening level wildlife risk analysis

Risk to piscivorous wildlife was evaluated with models based on adult dietary exposure or tissue concentrations. Dietary-based toxicity reference values (TRVs) based on no observed adverse effect levels (NOAELs) from the scientific literature were used for most contaminants (Linder et al. 1980; Hill and Camardese 1986; Sample et al. 1996; Tillitt et al. 1996; USEPA 1995). An adjustment factor for differences in body size was applied when the mammal NOAEL TRV was not based on a mink study; a scaling factor of 1 was used for interspecies extrapolation among birds (Sample et al. 1996). Consensus TRVs adopted by the USEPA were used for arsenic, cadmium, chromium, copper, nickel, and lead (USEPA 1995; 2005a, b, c, d; 2007a, b). Tissuebased TRVs developed from NOAEL egg concentrations were used for total DDT, total PCB, and TCDD-EQ in the bald eagle model because developing embryos are more sensitive to these contaminants than adult birds (Elliott and Harris 2001). The TRV for TCDD-EQ was based on a toxic equivalent that was developed using consensus toxic equivalent factors (van den Berg et al. 1998; Elliott and Harris 2001). A tissue-based TRV was also used for mercury in the bald eagle model (Giesy et al. 1995) after the use of a dietary-based TRV $(0.0064 \mathrm{mg} / \mathrm{kg} / \mathrm{d}$; Sample 


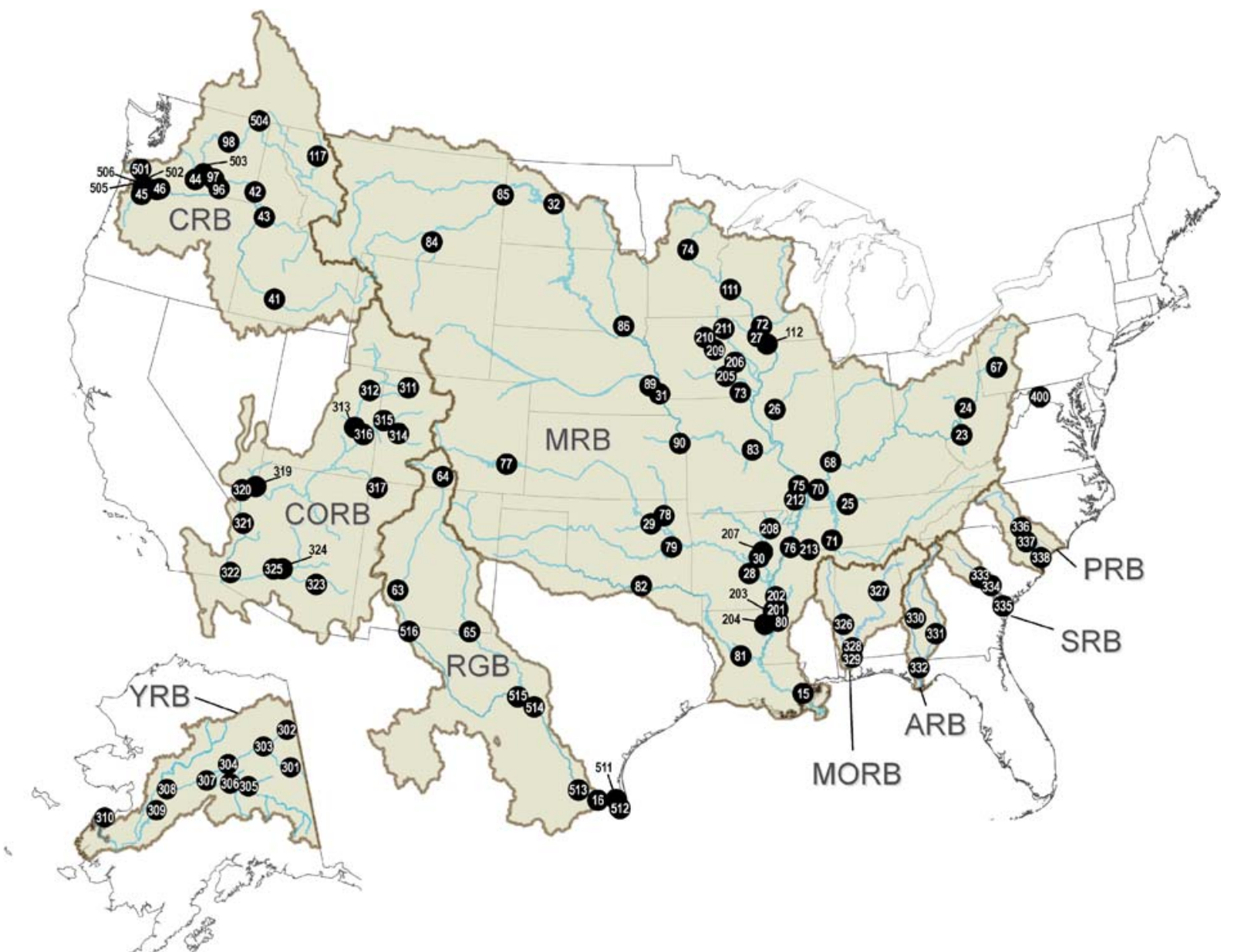

Fig. 1 Freshwater fish collection sites in the Yukon River Basin $(Y R B)$, Columbia River Basin $(C R B)$, Colorado River Basin $(C O R B)$, Rio Grande Basin $(R G B)$, Mississippi River Basin $(M R B)$, Mobile
River Basin $(M O R B)$, Apalachicola River Basin $(A R B)$, Savannah River Basin $(S R B)$, and Pee Dee River Basin $(P R B)$. See Table 1 for specific site information

Table 2 Benthivorous and piscivorous species collected from each basin

\begin{tabular}{|c|c|c|}
\hline River Basin & Benthivores & Piscivores \\
\hline Yukon & Longnose sucker & Northern pike, burbot \\
\hline Columbia & Common carp, largescale sucker & $\begin{array}{l}\text { Largemouth bass, smallmouth bass, northern pikeminnow, walleye, } \\
\text { rainbow trout }\end{array}$ \\
\hline Colorado & Common carp, white sucker & Largemouth bass, smallmouth bass, channel catfish, brown trout \\
\hline Rio Grande & Common carp & $\begin{array}{l}\text { Largemouth bass, smallmouth bass, spotted bass, white bass, } \\
\text { northern pike, channel catfish }\end{array}$ \\
\hline Mississippi & $\begin{array}{l}\text { Common carp, white sucker, } \\
\text { smallmouth buffalo }\end{array}$ & $\begin{array}{l}\text { Largemouth bass, smallmouth bass, spotted bass, striped bass, } \\
\text { white bass, northern pike, goldeye, sauger, brown trout }\end{array}$ \\
\hline Mobile & Common carp & Largemouth bass \\
\hline Apalachicola & Common carp & Largemouth bass \\
\hline Savannah & Common carp, redhorse sucker & Largemouth bass \\
\hline Pee Dee & Common carp & Largemouth bass \\
\hline
\end{tabular}


Table 3 Detection limits ( $\mu \mathrm{g} / \mathrm{g}$ wet weight unless otherwise noted) for organochlorine residues

\begin{tabular}{|c|c|c|c|c|c|}
\hline Analyte & $\begin{array}{l}\text { Mississippi River } \\
\text { Basin (1995) }\end{array}$ & $\begin{array}{l}\text { Rio Grande and } \\
\text { Columbia River } \\
\text { Basins (1997) }\end{array}$ & $\begin{array}{l}\text { Yukon River } \\
\text { Basin (2002) }\end{array}$ & $\begin{array}{l}\text { Colorado River } \\
\text { Basin (2003) }\end{array}$ & $\begin{array}{l}\text { Mobile, Apalachicola, } \\
\text { Savannah, and Pee Dee } \\
\text { River Basins (2004) }\end{array}$ \\
\hline Pentachlorobenzene & NM & NM & $0.00005-0.00006$ & 0.00007 & 0.00007 \\
\hline Hexachlorobenzene & 0.01 & 0.01 & $0.0021-0.0025$ & 0.00026 & NA \\
\hline Pentachloroanisole & NM & NM & $0.00023-0.00025$ & 0.00013 & 0.00010 \\
\hline$\alpha-\mathrm{HCH}$ & 0.01 & 0.01 & $0.00018-0.00020$ & 0.00008 & 0.00010 \\
\hline$\beta-\mathrm{HCH}$ & 0.01 & 0.01 & $0.00017-0.00019$ & 0.00019 & 0.00030 \\
\hline$\gamma-\mathrm{HCH}$ & 0.01 & 0.01 & $0.00039-0.00044$ & 0.00051 & 0.00010 \\
\hline$\delta-\mathrm{HCH}$ & 0.01 & 0.01 & $0.00010-0.00011$ & 0.00005 & 0.00010 \\
\hline Aldrin & NM & NM & NA & 0.00027 & 0.00009 \\
\hline Dieldrin & 0.01 & 0.01 & 0.00016 & 0.00015 & 0.00008 \\
\hline Endrin & 0.01 & 0.01 & $0.00011-0.00012$ & 0.00010 & 0.00025 \\
\hline Dacthal & $\mathrm{NM}$ & NM & $0.00029-0.00032$ & 0.00050 & 0.00013 \\
\hline Heptachlor & NM & NM & $0.00019-0.00021$ & 0.00019 & 0.00010 \\
\hline Heptachlor epoxide & 0.01 & 0.01 & $0.00019-0.00021$ & 0.00001 & 0.00010 \\
\hline Oxychlordane & 0.01 & 0.01 & $0.00008-0.00009$ & 0.00008 & 0.00010 \\
\hline cis-chlordane & 0.01 & 0.01 & $0.00019-0.00022$ & 0.00004 & 0.00028 \\
\hline trans-chlordane & 0.01 & 0.01 & $0.00031-0.00035$ & 0.00023 & NA \\
\hline cis-nonachlor & 0.01 & 0.01 & $0.00008-0.00009$ & 0.00010 & NA \\
\hline trans-nonachlor & 0.01 & 0.01 & $0.00021-0.00023$ & NA & NA \\
\hline$o, p$-DDE & 0.01 & 0.01 & $0.00015-0.00016$ & 0.00008 & 0.00081 \\
\hline$o, p^{\prime}-\mathrm{DDD}$ & 0.01 & 0.01 & $0.00061-0.00070$ & 0.00055 & 0.00010 \\
\hline$o, p^{\prime}$-DDT & 0.01 & 0.01 & $0.00012-0.00013$ & 0.00053 & 0.00010 \\
\hline$p, p$ '-DDE & 0.01 & 0.01 & $0.00039-0.00042$ & NA & NA \\
\hline$p, p '-\mathrm{DDD}$ & 0.01 & 0.01 & $0.00038-0.00043$ & 0.00067 & NA \\
\hline$p, p '-\mathrm{DDT}$ & 0.01 & 0.01 & $0.0014-0.0016$ & 0.0014 & 0.00047 \\
\hline Endosulfan I & NM & NM & $0.00034-0.00039$ & 0.00021 & 0.00010 \\
\hline Endosulfan II & $\mathrm{NM}$ & NM & $0.00014-0.00019$ & 0.00017 & 0.00010 \\
\hline Endosulfan sulfate & $\mathrm{NM}$ & NM & $0.00079-0.00090$ & 0.00005 & 0.00014 \\
\hline Methoxychlor & $\mathrm{NM}$ & NM & $0.0017-0.0019$ & 0.00035 & 0.00010 \\
\hline Mirex & 0.01 & 0.01 & 0.00001 & 0.00005 & 0.00010 \\
\hline PCB & 0.05 & 0.03 & 0.02 & 0.048 & NA \\
\hline Toxaphene & 0.05 & 0.03 & 0.011 & 0.024 & 0.010 \\
\hline TCDD-EQ (pg/g) & $0.2-1.0$ & $0.4-1.3$ & 1.7 & $0.1-0.5$ & $1.0-2.6$ \\
\hline
\end{tabular}

Study year is shown in parentheses

$H C H$ Hexachlorocyclohexane, $P C B$ polychlorinated biphenyl, $N M$ analyte not measured, $N A$ all concentrations were greater than the detection limit

et al. 1996) was found to be too conservative, resulting in risk at most sites $(n=105)$. Models assuming a diet of $100 \%$ fish were used to screen for risk. Site-specific data for other exposure pathways such as water and sediment were not available for all sites and were not considered in this risk evaluation; exposure from these other pathways was expected to be low and therefore would contribute little to the overall risk. A no effects hazard concentration (NEHC) was calculated for each spe- cies modeled using the equation NEHC $(\mu \mathrm{g} / \mathrm{g})=$ [dietary-based NOAEL TRV (mg/kg body weight/ day)]/food ingestion rate $(\mathrm{kg}$ food/ $\mathrm{kg}$ body weight/ day)] or [tissue-based NOAEL TRV (mg/kg egg)/ biomagnification factor $\left.(\mathrm{BMF})_{\text {fish } \rightarrow \text { egg }}\right]$. The bald eagle-specific BMFs were 28 for total PCBs, 19 for TCDD-EQ, 22 for total DDT, and one for mercury (Giesy et al. 1995). Piscivorous wildlife may be at risk from a contaminant if the measured concentration in the fish composite sample exceeds the NEHC. Risk 
Table 4 Detection limits ( $\mu \mathrm{g} / \mathrm{g}$ wet weight) for elemental contaminants

\begin{tabular}{llllll}
\hline Analyte & $\begin{array}{l}\text { Mississippi River } \\
\text { Basin (1995) }\end{array}$ & $\begin{array}{l}\text { Rio Grande and Columbia } \\
\text { River Basins (1997) }\end{array}$ & $\begin{array}{l}\text { Yukon River } \\
\text { Basin (2002) }\end{array}$ & $\begin{array}{l}\text { Colorado River } \\
\text { Basin (2003) }\end{array}$ & $\begin{array}{l}\text { Mobile, Apalachicola, Savannah, } \\
\text { and Pee Dee River Basins (2004) }\end{array}$ \\
\hline Arsenic & $0.11-0.27$ & $0.03-0.32$ & NA & NA & 0.01 \\
Cadmium & $0.02-0.05$ & $0.02-0.06$ & $0.02-0.03$ & $0.02-0.03$ & 0.01 \\
Chromium & 0.16 & NA & $0.22-0.27$ & $0.24-0.35$ & NA \\
Copper & NA & NA & NA & NA & NA \\
Mercury & $0.04-0.05$ & $0.02-0.06$ & NA & NA & NA \\
Nickel & $0.16-0.26$ & $0.22-0.33$ & $0.21-0.27$ & $0.24-0.35$ & 0.01 \\
Lead & $0.01-0.04$ & $0.03-0.14$ & $0.21-0.27$ & $0.24-0.35$ & 0.01 \\
Selenium & $0.12-0.18$ & $0.22-0.27$ & NA & NA & NA \\
Zinc & NA & NA & NA & NA & NA \\
\hline
\end{tabular}

Study year is shown in parentheses

$N A$ All concentrations were greater than the detection limit

was categorized as none $(<\mathrm{NEHC})$, low $(1-10 \times$ $\mathrm{NEHC})$, or high $(>10 \times \mathrm{NEHC})$. Model species considered in the risk analysis included the bald eagle as an avian receptor and mink as a mammalian receptor. Both of these species have distributions that span most of the United States and consume the large, adult fish such as those collected by the BEST-LRMN Project. Some contaminants we evaluated have been associated with the death of bald eagles and mink. For example, reproductive impairment of mink has been directly related to PCBs in Great Lakes fish (Aulerich and Ringer 1977; Heaton et al. 1995), and mercury has been implicated in increased mink mortality (Wren et al. 1987). Bald eagle survival and reproduction have been negatively affected by dieldrin, $p, p^{\prime}-$ DDE, and PCBs in the environment (Reichel et al. 1984; Wiemeyer et al. 1984). Benthivorous and piscivorous fish were examined separately because fish in higher trophic levels (piscivorous fish) generally have higher concentrations of accumulative contaminants such as total PCBs and mercury, but benthivorous fish species are the preferred prey in some bald eagle populations (Haywood and Ohmart 1986).

\section{Results}

Environmental contaminant concentrations

Organochlorine concentrations were less than the detection limits in most samples (Table 5). Transnonachlor, $p, p^{\prime}$-DDE, $p, p^{\prime}$-DDD, total $\mathrm{PCBs}$, and TCDD-EQ were the most frequently detected com- pounds. Higher detection limits for the Mississippi, Rio Grande, and Columbia River Basins than for those other basins sampled influenced mean and median concentrations of many organochlorine residues (Table 5). Mean concentrations ranged from 0.00007 to $0.08089 \mu \mathrm{g} / \mathrm{g}$, and median concentration ranged from 0.00007 to $0.0723 \mu \mathrm{g} / \mathrm{g}$ (most $<0.01 \mu \mathrm{g} /$ g). Mean and median concentrations were greatest for total chlordanes, $p, p^{\prime}$-DDE, total DDT, total PCBs, and toxaphene (Table 5). The 85 th percentile ranged from $0.00021 \mu \mathrm{g} / \mathrm{g}$ for pentachlorobenzene and heptachlor to $0.450 \mu \mathrm{g} / \mathrm{g}$ for total PCBs and were $>0.02 \mu \mathrm{g} / \mathrm{g}$ for trans-nonachlor, total chlordanes, $p, p^{\prime}-$ DDE, $p, p^{\prime}$-DDD, total DDT, total PCBs, and toxaphene (Table 5). Maximum concentrations ranged from $0.00111 \mu \mathrm{g} / \mathrm{g}$ for aldrin to $11.37 \mu \mathrm{g} / \mathrm{g}$ for total DDT but were $<0.08 \mu \mathrm{g} / \mathrm{g}$ for most organochlorine residues (Table 5). The median TCDD-EQ concentration was $0.0000017 \mu \mathrm{g} / \mathrm{g}$ (Table 5). Mean concentrations of select organochlorine pesticides and total PCBs were less than those reported in fish from the historical NCBP (Table 5). Mean concentrations of Dacthal and heptachlor were tenfold lower than those measured in NCBP samples, and mean concentrations of chlordane components (oxychlordane, cis- and trans-chlordanes and nonachlors) also were lower in BEST-LRMN samples $(0.002-0.005 \mu \mathrm{g} / \mathrm{g})$ than NCBP samples $(0.007-0.011 \mu \mathrm{g} / \mathrm{g}$; Table 5). Mean $p, p^{\prime}$-homolog concentrations $(0.003-0.045 \mu \mathrm{g} / \mathrm{g})$ were lower than those reported in NCBP samples $(0.010$ $-0.070 \mu \mathrm{g} / \mathrm{g}$; Table 5). Concentrations of total PCBs and toxaphene also were lower in BEST-LRMN samples compared to those in NCBP samples. 
Table 5 Organochlorine chemical residue concentrations ( $\mu \mathrm{g} / \mathrm{g}$ wet weight) in whole-body fish composite samples from 1995 to 2004

\begin{tabular}{|c|c|c|c|c|c|c|}
\hline \multirow[t]{2}{*}{ Analyte } & \multicolumn{5}{|l|}{ 1995-2004 } & \multirow{2}{*}{$\frac{\text { NCBP }}{\text { Mean }}$} \\
\hline & No. $>$ DL/n & Mean & Median & 85 th & Maximum & \\
\hline Lipid (\%) & $409 / 409$ & 4.89 & 4.96 & 8.99 & 18.10 & N/A \\
\hline Pentachlorobenzene & $56 / 135$ & 0.00007 & 0.00007 & 0.00021 & 0.00119 & N/A \\
\hline Hexachlorobenzene & $110 / 409$ & 0.00277 & 0.01 & 0.01 & 0.068 & $<0.01$ \\
\hline Pentachloroanisole & $113 / 135$ & 0.00056 & 0.00057 & 0.00258 & 0.021 & $<0.01$ \\
\hline$\alpha-\mathrm{HCH}$ & $62 / 409$ & 0.00146 & 0.01 & 0.01 & 0.01 & 0.005 \\
\hline$\beta-\mathrm{HCH}$ & $55 / 409$ & 0.00192 & 0.01 & 0.01 & 0.01 & N/A \\
\hline$\gamma-\mathrm{HCH}$ & $40 / 409$ & 0.00178 & 0.01 & 0.01 & 0.01 & $<0.01$ \\
\hline$\delta-\mathrm{HCH}$ & $54 / 409$ & 0.00134 & 0.01 & 0.01 & 0.01 & N/A \\
\hline Aldrin & $38 / 135$ & 0.00012 & 0.00019 & 0.00027 & 0.00111 & N/A \\
\hline Dieldrin & $198 / 409$ & 0.00411 & 0.01 & 0.017 & 0.250 & 0.008 \\
\hline Endrin & $76 / 409$ & 0.00215 & 0.01 & 0.01 & 0.71 & 0.005 \\
\hline Dacthal & $46 / 135$ & 0.00025 & 0.00032 & 0.00070 & 0.00928 & 0.007 \\
\hline Heptachlor & $14 / 135$ & 0.00009 & 0.00019 & 0.00021 & 0.00382 & 0.007 \\
\hline Heptachlor epoxide & $115 / 409$ & 0.00189 & 0.01 & 0.01 & 0.075 & N/A \\
\hline Oxychlordane & $116 / 409$ & 0.00229 & 0.01 & 0.01 & 0.028 & 0.007 \\
\hline cis-chlordane & $175 / 409$ & 0.00374 & 0.01 & 0.013 & 0.120 & 0.011 \\
\hline trans-chlordane & $150 / 409$ & 0.00307 & 0.01 & 0.01 & 0.35 & 0.008 \\
\hline cis-nonachlor & $172 / 409$ & 0.00351 & 0.01 & 0.01 & 0.047 & 0.009 \\
\hline trans-nonachlor & $229 / 409$ & 0.00548 & 0.01 & 0.02 & 0.31 & 0.013 \\
\hline ¿Chlordane & N/A & 0.0236 & 0.03 & 0.057 & 0.529 & N/A \\
\hline$o, p '-\mathrm{DDE}$ & $89 / 409$ & 0.00238 & 0.01 & 0.01 & 0.022 & N/A \\
\hline$o, p '-\mathrm{DDD}$ & $99 / 409$ & 0.00283 & 0.01 & 0.01 & 0.34 & $\mathrm{~N} / \mathrm{A}$ \\
\hline$o, p^{\prime}$-DDT & $67 / 409$ & 0.00189 & 0.01 & 0.01 & 0.24 & N/A \\
\hline$p, p '-\mathrm{DDE}$ & $382 / 409$ & 0.0447 & 0.05 & 0.29 & 8.30 & 0.070 \\
\hline$p, p$ '-DDD & $250 / 409$ & 0.00907 & 0.01 & 0.04 & 2.80 & 0.019 \\
\hline$p, p^{\prime}-\mathrm{DDT}$ & $88 / 409$ & 0.00332 & 0.01 & 0.01 & 0.31 & 0.010 \\
\hline EDDT & $\mathrm{N} / \mathrm{A}$ & 0.0809 & 0.07 & 0.38 & 11.37 & $0.110^{\mathrm{a}}$ \\
\hline Endosulfan I & $13 / 135$ & 0.00011 & 0.00021 & 0.00037 & 0.00390 & N/A \\
\hline Endosulfan II & $47 / 135$ & 0.00015 & 0.00017 & 0.00050 & 0.05400 & N/A \\
\hline Endosulfan sulfate & $80 / 135$ & 0.00054 & 0.00079 & 0.00220 & 0.07900 & N/A \\
\hline Methoxychlor & $15 / 135$ & 0.00021 & 0.00035 & 0.00180 & 0.00960 & N/A \\
\hline Mirex & $118 / 409$ & 0.00183 & 0.01 & 0.01 & 0.08 & 0.006 \\
\hline PCB & $256 / 409$ & 0.084 & 0.067 & 0.450 & 3.30 & 0.335 \\
\hline Toxaphene & $83 / 409$ & 0.03 & 0.05 & 0.05 & 0.83 & 0.066 \\
\hline TCDD-EQ & $255 / 409$ & 0.0000015 & 0.0000017 & 0.000007 & 0.0000680 & N/A \\
\hline
\end{tabular}

Number of samples with a concentration greater than the detection limit (DL), geometric mean, median, and 85th percentile concentrations are presented. The geometric mean from 1986 National Contaminant Biomonitoring Program (NCBP) samples also is provided for comparison (Schmitt et al. 1999). $\Sigma$ Chlordane is the sum of heptachlor epoxide, oxychlordane, cis-chlordane, transchlordane, cis-nonachlor, and trans-nonachlor, and EDDT is the sum of $o, p^{\prime}-$ and $p, p^{\prime}$-DDE, DDD, and DDT. Censored values were represented by one half the detection limit in the summed values

$N / A$ not applicable

Elemental contaminant concentrations exceeded detection limits in most samples (Table 6). Mean concentrations ranged from $0.03 \mu \mathrm{g} / \mathrm{g}$ for cadmium to $35.2 \mu \mathrm{g} / \mathrm{g}$ for zinc, and median concentrations ranged from 0.04 to $36.0 \mu \mathrm{g} / \mathrm{g}$ (Table 6). The 85 th percentile was $<1.0 \mu \mathrm{g} / \mathrm{g}$ for arsenic, cadmium, mercury, nickel, lead, and selenium and $>1.0 \mu \mathrm{g} / \mathrm{g}$ for chromium, copper, and zinc. Maximum concentrations ranged from $0.51 \mu \mathrm{g} / \mathrm{g}$ for cadmium to $150 \mu \mathrm{g} / \mathrm{g}$ for zinc but were $<10 \mu \mathrm{g} / \mathrm{g}$ for most elemental contaminants (Table 6). Mean concentrations of arsenic, cadmium, copper, mercury, lead, selenium, and zinc were greater 
Table 6 Elemental contaminant concentrations ( $\mu \mathrm{g} / \mathrm{g}$ wet weight) in whole-body fish composite samples from 1995 to 2004

\begin{tabular}{|c|c|c|c|c|c|c|c|}
\hline \multirow[t]{2}{*}{ Analyte } & \multicolumn{5}{|l|}{ 1995-2004 } & \multicolumn{2}{|c|}{ NCBP } \\
\hline & No. $>$ DL $/ n$ & Mean & Median & 85 th & Maximum & Mean & 85 th \\
\hline Moisture (\%) & $409 / 409$ & 72.2 & 72.6 & 75.2 & 79.9 & N/A & N/A \\
\hline Arsenic & $233 / 409$ & 0.11 & 0.19 & 0.27 & 1.95 & 0.08 & 0.24 \\
\hline Cadmium & $180 / 409$ & 0.03 & 0.04 & 0.09 & 0.51 & 0.01 & 0.04 \\
\hline Chromium & $393 / 409$ & 0.76 & 0.60 & 2.38 & 71.8 & N/A & N/A \\
\hline Copper & $409 / 409$ & 0.80 & 0.86 & 1.26 & 3.92 & 0.79 & 1.7 \\
\hline Mercury & $409 / 409$ & 0.13 & 0.13 & 0.27 & 0.78 & 0.09 & 0.18 \\
\hline Nickel & $274 / 409$ & 0.30 & 0.30 & 0.73 & 5.59 & N/A & N/A \\
\hline Lead & $233 / 409$ & 0.07 & 0.10 & 0.27 & 9.29 & 0.06 & 0.21 \\
\hline Selenium & $398 / 409$ & 0.59 & 0.53 & 0.98 & 4.66 & 0.42 & 0.66 \\
\hline Zinc & $409 / 409$ & 35.2 & 36.0 & 76.0 & 150 & 21.2 & 31.7 \\
\hline
\end{tabular}

Number of samples with a concentration greater than the detection limit (DL), geometric mean, median, and 85th percentile concentrations are presented. The geometric mean and 85th percentiles from 1986 NCBP samples are provided for comparison (Schmitt et al. 1999)

$N / A$ Not applicable

than those reported in fish from the historical NCBP (Table 6). Most differences in mean concentrations between BEST-LRMN samples and NCBP samples were $<0.04 \mu \mathrm{g} / \mathrm{g}$, but mean differences for selenium $(0.17 \mu \mathrm{g} / \mathrm{g})$ and zinc $(14.0 \mu \mathrm{g} / \mathrm{g})$ were greater (Table 6). The 85 th percentile concentrations of arsenic, cadmium, mercury, lead, selenium, and zinc also were greater than those reported in fish from the NCBP (Table 6). Differences in mean concentrations of arsenic, cadmium, mercury, and lead were $<0.10 \mu \mathrm{g} / \mathrm{g}$, but differences in copper $(0.44 \mu \mathrm{g} / \mathrm{g})$, selenium $(0.32 \mu \mathrm{g} / \mathrm{g})$, and zinc $(54.8 \mu \mathrm{g} / \mathrm{g})$ were greater (Table 6$)$.

Toxicity thresholds

Concentrations in whole-body fish samples exceeded literature-based toxicity thresholds for dieldrin, total chlordane, total DDT, total PCBs, toxaphene, TCDD$\mathrm{EQ}$, cadmium, chromium, mercury, lead, selenium, and zinc (Table 7). Concentrations of hexachlorobenzene, arsenic, and copper did not exceed toxicity thresholds for fish and wildlife at any sampling location. Toxicity thresholds meeting our criteria were not found for pentachlorobenzene, pentachloroanisole, $\alpha$-hexachlorocyclohexane $(\mathrm{HCH}), \beta-\mathrm{HCH}, \delta-\mathrm{HCH}$, aldrin, endrin, Dacthal, endosulfans, methoxychlor, mirex, or nickel (Table 7). Concentrations of some contaminants differed between trophic levels. Concentrations of dieldrin, endrin, total chlordane, total DDT, total PCBs, toxaphene, TCDD-EQ, chromium, and selenium generally were similar in benthivorous and piscivorous fish (Fig. 2). Concentrations of endrin, total DDT, and toxaphene in Mississippi River Basin samples were greater in benthivores compared to piscivores (Fig. 2). Concentrations of cadmium, lead, and zinc were greater in benthivores than in piscivores, but mercury was greater in piscivorous fish than in benthivorous fish (Fig. 2).

The greatest concentrations and therefore the greatest exceedences of toxicity thresholds were found in Mississippi River Basin samples. Toxicity thresholds for dieldrin and total chlordane were exceeded by only a few samples from the Mississippi River Basin (Fig. 2); carp samples from Sites 76 and 206 exceeded thresholds for both of these contaminants. Total DDT concentrations exceeded toxicity thresholds in 126 samples representing all basins except the Yukon, Savannah, and Pee Dee River Basins and were greatest in samples from the Columbia, Colorado, Rio Grande, and Mississippi River Basins (Fig. 2). A total of 172 samples representing all basins except the Rio Grande and Yukon River Basins exceeded protective thresholds for total PCBs. Total PCB concentrations were greatest in samples from the Columbia, Colorado, Mississippi, Mobile, and Apalachicola River Basins (Fig. 2). Toxaphene concentrations exceeded toxicity thresholds in 42 samples representing all basins except the Savannah River Basin but were generally greatest in samples from the Columbia, Rio Grande, and Mississippi River Basins (Fig. 2). TCDD-EQ concentrations exceeded toxicity thresholds for fish 
Table 7 Toxicity thresholds for fish and wildlife and the number of samples and sites that exceed these concentrations ${ }^{\mathrm{a}}$

\begin{tabular}{|c|c|c|c|c|}
\hline \multirow[t]{2}{*}{ Contaminant } & \multicolumn{4}{|l|}{ Toxicity threshold } \\
\hline & Conc. $(\mu \mathrm{g} / \mathrm{g})$ & Reference & No. of samples ${ }^{b}$ & No. of sites ${ }^{b}$ \\
\hline Hexachlorobenzene & 0.33 & Newell et al. 1987 & 0 & 0 \\
\hline$\gamma-\mathrm{HCH}$ & 0.1 & Newell et al. 1987 & 0 & 0 \\
\hline Dieldrin & $0.12-5.65$ & $\begin{array}{l}\text { Newell et al. 1987; Shubat \& Curtis 1986; } \\
\text { Peakall } 1996\end{array}$ & 5 & 2 \\
\hline ¿Chlordane & 0.30 & Eisler 1990 & 5 & 2 \\
\hline इDDT & $0.15-3.0$ & $\begin{array}{l}\text { Anderson et al. 1975; Blus 1996; } \\
\text { Beckvar et al. } 2005\end{array}$ & 126 & 42 \\
\hline PCB & $0.11-0.48$ & Hornshaw et al. 1983; Newell et al. 1987 & 172 & 54 \\
\hline Toxaphene & $0.035-0.90$ & Mayer et al. 1975 & 42 & 18 \\
\hline TCDD-EQ & $0.0000044-0.00003$ & $\begin{array}{l}\text { Nosek et al. 1992; Heaton et al. 1995; } \\
\text { Tillitt et al. 1996; Walker et al. } 1996\end{array}$ & 108 & 49 \\
\hline Arsenic & $2.2-11.6$ & Gilderhus 1966; McGeachy and Dixon 1992 & 0 & 0 \\
\hline Cadmium & $0.23-15.6$ & Spehar 1976; Eisler 1985; Hansen et al. 2002 & 17 & 12 \\
\hline Chromium & 1.0 & Eisler 1986 & 121 & 59 \\
\hline Copper & $11.1-42.0$ & Stouthart et al. 1996 & 0 & 0 \\
\hline Mercury & $0.1-0.3$ & $\begin{array}{l}\text { Barr 1986; Yeardley et al. 1998; } \\
\text { Beckvar et al. 2005; }\end{array}$ & 278 & 99 \\
\hline Lead & $0.4-8.8$ & Holcombe et al. 1976 & 12 & 9 \\
\hline Selenium & $0.75-1.0$ & Lemly 1996; Hamilton 2004 & 101 & 43 \\
\hline Zinc & $40-64$ & Spehar 1976 & 198 & 97 \\
\hline
\end{tabular}

Toxicity thresholds for dieldrin, toxaphene, arsenic, cadmium, lead, selenium, and zinc were based on whole-body fish concentrations. Toxicity thresholds for hexachlorobenzene, $\gamma-\mathrm{HCH}, \Sigma \mathrm{Chlordane,} \mathrm{EDDT}, \mathrm{PCB}$, TCDD-EQ, and chromium were based on wildlife toxicity values. Toxicity thresholds were not available for pentachlorobenzene, pentachloroanisole, $\alpha-\mathrm{HCH}, \beta-\mathrm{HCH}, \delta-\mathrm{HCH}$, aldrin, endrin, Dacthal, endosulfans, methoxychlor, mirex, and nickel. $\Sigma$ Chlordane is the sum of heptachlor epoxide, oxychlordane, cischlordane, trans-chlordane, cis-nonachlor, and trans-nonachlor, and $\mathrm{EDDT}$ is the sum of $o, p^{\prime}-$ and $p, p^{\prime}$-DDE, DDD, and DDT

${ }^{\text {a }}$ Number of samples $=409$ for all contaminants except for TCDD-EQ $(n=408)$. Number of sites $=111$

${ }^{b}$ The number of samples or sites to exceed lowest toxicity threshold available for a contaminant

and wildlife in 108 samples representing all basins except the Yukon and Savannah River Basins and were greatest in samples from the Columbia, Mississippi, and Mobile River Basins (Fig. 2). Cadmium concentrations were generally greater in carp and other benthivores than in piscivores and exceeded toxicity thresholds in 17 samples representing the Columbia, Colorado, and Mississippi River Basins (Fig. 2). Conversely, mercury concentrations were consistently greater in piscivores and exceeded toxicity thresholds in 278 samples representing all basins (Fig. 2). Protective toxicity thresholds for lead were exceeded by 12 samples representing the Columbia, Rio Grande, Mississippi, and Apalachicola River Basins, and concentrations were greatest in benthivore samples from the Columbia River Basin (Fig. 2). Selenium concentrations exceeded toxicity thresholds in 101 samples representing all basins except the Savannah and Pee Dee River Basins and were greatest in samples from the Colorado, Rio Grande, and Mississippi River Basins (Fig. 2). Zinc concentrations were generally greater in carp and other benthivores than piscivores, but concentrations in northern pike were greater than longnose sucker from the Yukon River Basin. Zinc exceeded toxicity thresholds in 198 samples representing all basins (Fig. 2).

Screening level wildlife risk analysis

Concentrations of dieldrin, endrin, total DDT, total PCBs, and TCDD-EQ in whole-body fish samples represented a risk to piscivorous wildlife at one or more sites. No risk of dieldrin and endrin exposure to bald eagle and mink was found at most sites. Dieldrin concentrations at two Mississippi River Basin sites (Sites 76 and 206; $0.15-0.25 \mu \mathrm{g} / \mathrm{g}$ ) exceeded the NEHC for mink (Figs. 2 and 3), and endrin concentrations in samples from Site $76(0.22-0.71 \mu \mathrm{g} / \mathrm{g})$ also 

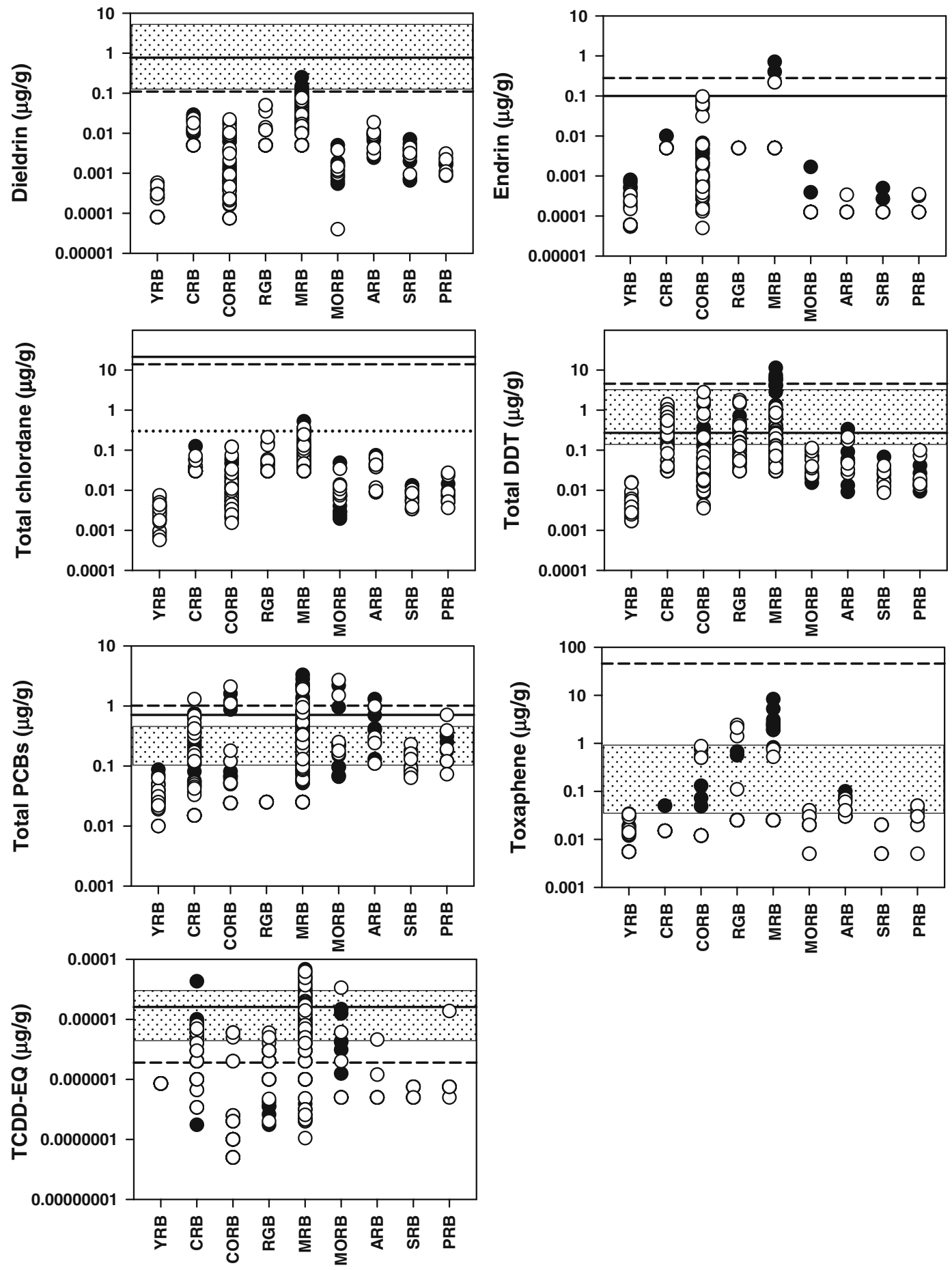

Fig. 2 Organochlorine residue and elemental concentrations (all $\mu \mathrm{g} / \mathrm{g}$ wet-weight) in whole-body composite samples of benthivorous (black circles) and piscivorous (white circles) fish from the Yukon River Basin $(Y R B)$, Columbia River Basin $(C R B)$, Colorado River Basin (CORB), Rio Grande Basin $(R G B)$, Mississippi River Basin $(M R B)$, Mobile River Basin $(M O R B)$, Apalachicola River Basin $(A R B)$, Savannah River

Basin $(S R B)$, and Pee Dee River Basin (PRB). Literature-based toxicity thresholds are represented by dotted lines or boxes (see Table 7 for reference). No effect hazard concentrations (NEHCs) for bald eagle (solid line) and mink (dashed line) are presented for each contaminant (see Table 8 for references and text for calculation) 

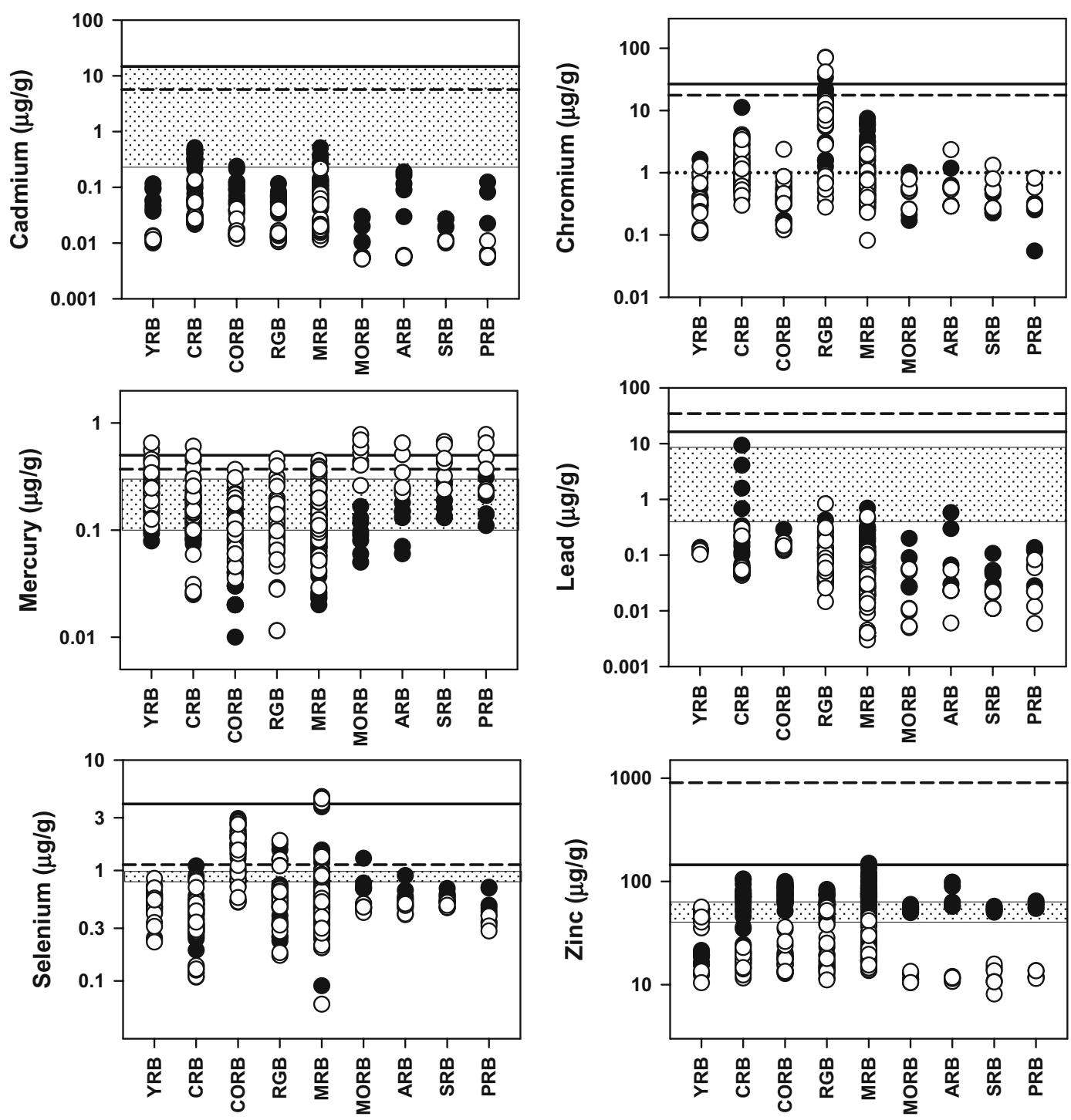

Fig. 2 (continued)

exceeded the NEHC for bald eagle and mink (Fig. 2). Total DDT concentrations did not represent a risk to bald eagle at sites from the Yukon, Mobile, Savannah, and Pee Dee River Basins (Fig. 3). Total DDT concentrations exceeded the NEHC for bald eagle in samples from the Columbia River Basin (34 samples from 12 sites; 0.27-1.41 $\mu \mathrm{g} / \mathrm{g}$ ), Colorado River Basin (nine samples from three sites; $0.28-2.80 \mu \mathrm{g} / \mathrm{g}$ ), Rio Grande Basin (12 samples from four sites; 0.30 $-1.78 \mu \mathrm{g} / \mathrm{g}$ ), Mississippi River Basin (36 samples from 16 sites; $0.27-11.37 \mu \mathrm{g} / \mathrm{g}$ ), and Apalachicola River Basin (one sample from Site 330; $0.33 \mu \mathrm{g} / \mathrm{g}$; Figs. 2 and 3). The risk of total DDT to mink was lower with few NEHC exceedences. Carp from Sites
201, 202, 203, and 204 in the Mississippi River Basin $(5.49-11.37 \mu \mathrm{g} / \mathrm{g})$ were the only samples with total DDT concentrations that exceeded the NEHC for mink (Figs. 2 and 3). Total PCB concentrations did not represent a risk to piscivorous wildlife at sites in the Yukon, Rio Grande, Savannah, and Pee Dee River Basins (Fig. 3). Total PCB concentrations exceeded the NEHC for bald eagle in samples from the Columbia River Basin (two samples from two sites; 0.75-1.3 $\mu \mathrm{g} / \mathrm{g}$ ), Colorado River Basin (six sample from two sites; 0.87-2.1 $\mu \mathrm{g} / \mathrm{g}$ ), Mississippi River Basin (20 samples from nine sites; 0.75-3.3 $\mu \mathrm{g} / \mathrm{g}$ ), Mobile River Basin (four samples from Site 327; 0.95-2.7 $\mu \mathrm{g} / \mathrm{g}$ ), and Apalachicola River Basin (three 
samples from Site 330; 0.94-1.3 $\mu \mathrm{g} / \mathrm{g}$; Figs. 2 and 3). The risk of total PCB exposure was lower to mink than bald eagle (Fig. 3). Concentrations of total PCBs exceeded the NEHC for mink in samples from the Columbia River Basin (one sample from Site 503; $1.3 \mu \mathrm{g} / \mathrm{g}$ ), Colorado River Basin (four samples from two sites; 1.1-2.1 $\mu \mathrm{g} / \mathrm{g}$ ), Mississippi River Basin (12 samples from five sites; $1.1-3.3 \mu \mathrm{g} / \mathrm{g}$ ), Mobile River Basin (three samples from Site 327; $1.5-2.7 \mu \mathrm{g} / \mathrm{g}$ ), and Apalachicola River Basin (one sample from Site 330; $1.3 \mu \mathrm{g} / \mathrm{g}$; Figs. 2 and 3). No risk of TCDD-EQ exposure to bald eagle and mink was found at sites in the Yukon or Savannah River Basins (Fig. 3). TCDDEQ concentrations exceeded the NEHC for bald eagle in samples from the Columbia River Basin (one sample from Site 96; $0.000043 \mu \mathrm{g} / \mathrm{g}$ ), Mississippi River Basin (29 samples from 18 sites; 0.000017$0.000068 \mu \mathrm{g} / \mathrm{g}$ ), and Mobile River Basin (one sample from Site 327; $0.000034 \mu \mathrm{g} / \mathrm{g}$; Figs. 2 and 3). The risk of TCDD-EQ exposure was greater to mink than bald eagle (Fig. 3). TCDD-EQ concentrations exceeded the NEHC for mink in samples from the Columbia River Basin (38 samples from 15 sites; 0.000002-0.000043 $\mu \mathrm{g} / \mathrm{g}$ ), Colorado River Basin (six samples from three sites; 0.000002-0.000006 $\mu \mathrm{g} / \mathrm{g}$ ), Rio Grande Basin (25 samples from 10 sites; 0.000002-0.000006 $\mu \mathrm{g} / \mathrm{g}$ ), Mississippi River Basin (106 samples from 42 sites; $0.000002-0.000068 \mu \mathrm{g} / \mathrm{g}$ ), Mobile River Basin (eight samples from four sites; 0.000002-0.000034 $\mu \mathrm{g} / \mathrm{g}$ ), Apalachicola River Basin (one sample from Site 330; $0.0000046 \mu \mathrm{g} / \mathrm{g}$ ), and Pee Dee River Basin (one sample from Site 337; $0.000014 \mu \mathrm{g} / \mathrm{g}$; Figs. 2 and 3). A NOAEL TRV was not available for pentachlorobenzene, pentachloroanisole, aldrin, Dacthal, methoxychlor, mirex, or toxaphene in bald eagle and hexachlorobenzene, pentachloroanisole, Dacthal, and mirex in mink (Table 8); therefore, the risk of these contaminants is unknown.

Chromium, mercury, selenium, and zinc concentrations in whole-body fish samples represented a risk to piscivorous wildlife at one or more sites. Chromium represented a risk to piscivorous wildlife only in the Rio Grande Basin, where concentrations were comparatively high; they exceeded the NEHC for bald eagle, mink, or both in Rio Grande samples from Sites $63(71.8 \mu \mathrm{g} / \mathrm{g}), 511(34.0 \mu \mathrm{g} / \mathrm{g}), 512(18.3 \mu \mathrm{g} / \mathrm{g})$, $513(21.1-70.2 \mu \mathrm{g} / \mathrm{g})$, and $515(21.8 \mu \mathrm{g} / \mathrm{g}$; Figs. 2 and 4). No risk of mercury exposure to bald eagle was associated with samples from the Colorado, Rio
Grande, or Mississippi River Basins (Fig. 2). Mercury concentrations exceeded the NEHC for bald eagle in the Yukon River Basin (two samples from two sites; 0.56-0.65 $\mu \mathrm{g} / \mathrm{g}$ ), Columbia River Basin (one sample from Site 43; $0.61 \mu \mathrm{g} / \mathrm{g}$ ), Mobile River Basin (five samples from three sites; 0.52-0.78 $\mu \mathrm{g} / \mathrm{g}$ ), and Apalachicola-Flint-Chattahoochee River Basin (two samples from Site 332; 0.51-0.65 $\mu \mathrm{g} / \mathrm{g}$, Savannah River Basin (two samples from Site 334; 0.63$0.67 \mu \mathrm{g} / \mathrm{g}$ ), and Pee Dee River Basin (two samples from Site 338; $0.65-0.78 \mu \mathrm{g} / \mathrm{g}$; Figs. 2 and 4). For mink, samples from the Yukon River Basin (five samples from three sites; 0.37-0.65 $\mu \mathrm{g} / \mathrm{g}$ ), Columbia River Basin (six samples from four sites; 0.38$0.61 \mu \mathrm{g} / \mathrm{g}$ ), Rio Grande Basin (two samples from two sites; 0.40-0.46 $\mu \mathrm{g} / \mathrm{g}$ ), Mississippi River Basin (four samples from three sites; 0.39-0.45 $\mu \mathrm{g} / \mathrm{g}$ ), Mobile River Basin (seven samples from four sites; 0.40-0.78 $\mu \mathrm{g} / \mathrm{g}$ ), Apalachicola River Basin (two samples from Site 332; 0.51-0.65 $\mu \mathrm{g} / \mathrm{g}$ ), Savannah River Basin (four samples from three sites; 0.43$0.67 \mu \mathrm{g} / \mathrm{g}$ ), and Pee Dee River Basin (four samples from two sites; 0.37-0.78 $\mu \mathrm{g} / \mathrm{g}$ ) exceeded the NEHC for mercury (Figs. 2 and 4). Selenium did not represent a risk to piscivorous wildlife at any site in the Yukon, Columbia, Apalachicola, Savannah, or Pee Dee River Basins (Figs. 2 and 4). Selenium concentrations exceeded the NEHC for bald eagle at one site in the Mississippi River Basin (Site 77; 4.11-4.66 $\mu \mathrm{g} / \mathrm{g}$ ) and for mink in samples from the Colorado River Basin (34 samples from 12 sites; 1.29-2.95 $\mu \mathrm{g} / \mathrm{g}$ ), Rio Grande Basin (six samples from three sites; 1.24$1.87 \mu \mathrm{g} / \mathrm{g}$ ), Mississippi River Basin (ten samples from three sites; $1.18-4.66 \mu \mathrm{g} / \mathrm{g}$ ), and the Mobile River Basin (one sample from Site 329; $1.29 \mu \mathrm{g} / \mathrm{g}$; Figs. 2 and 4). No risk of zinc exposure to piscivorous wildlife was found at most sites; zinc concentrations in one Mississippi River Basin carp sample (Site $79 ; 150 \mu \mathrm{g} / \mathrm{g}$ ) represented a risk to bald eagle (Fig. 2). Concentrations of arsenic, cadmium, copper, nickel, and lead did not represent a risk to wildlife at any site.

\section{Discussion}

Overall, mean concentrations of organochlorine residues were less than historical NCBP concentrations; concentrations of arsenic, cadmium, copper, mercury, 


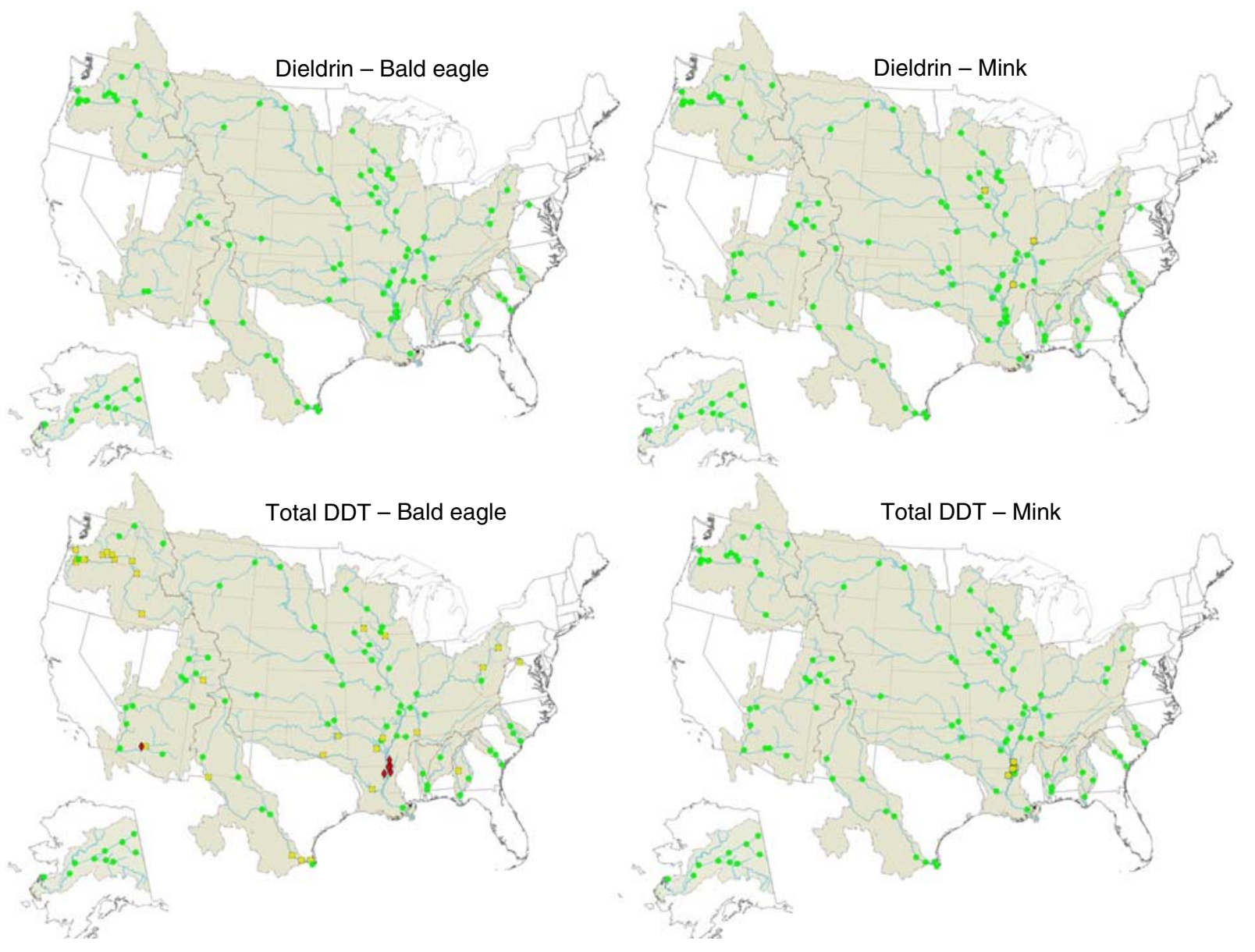

Fig. 3 Sites that exceed the no effects hazard concentration (NEHC) for selected organochlorine residues. See Fig. 1 for specific site locations and Table 8 for NEHC concentrations

lead, selenium, and zinc were greater than NCBP concentrations. Direct comparison of these datasets is precluded by collection location and species composition differences, which can bias mean concentrations. Concentrations of organochlorine pesticides and total PCBs were expected to decline because their use has been banned or regulated in the United States. However, high concentrations of organochlorine chemical residues remain evident in fish near point sources such as former manufacturing facilities and areas of high historical use. Elemental contaminants emanating from point sources have also been regulated, as have many pesticides containing potentially toxic trace elements. However, elemental contaminants also are natural constituents of the earth's crust; some are essential trace elements for terrestrial and aquatic organisms. In spite of regulation, elemental concentrations in fish remain elevated in some areas due to irrigated agriculture (Hamilton 2004), mining (Schmitt et al. 2006), and the combustion of fossil fuels (Baumann and Gillespie 1986; Yeardley et al. 1998), and both organochlorine and elemental contaminants are distributed globally by atmospheric transport. Considered independently, concentrations of most organochlorine residues and elemental contaminants we evaluated represented no or low risk to bald eagle and mink. Among the contaminants evaluated, the greatest risk to bald eagle and mink was associated with total DDT, total PCBs, TCDDEQ, mercury, and selenium. The risk of pentachloroanisole, aldrin, Dacthal, methoxychlor, mirex, or toxaphene to bald eagle or mink was unknown; NEHCs could not be calculated because NOAELS were not available for these compounds. 

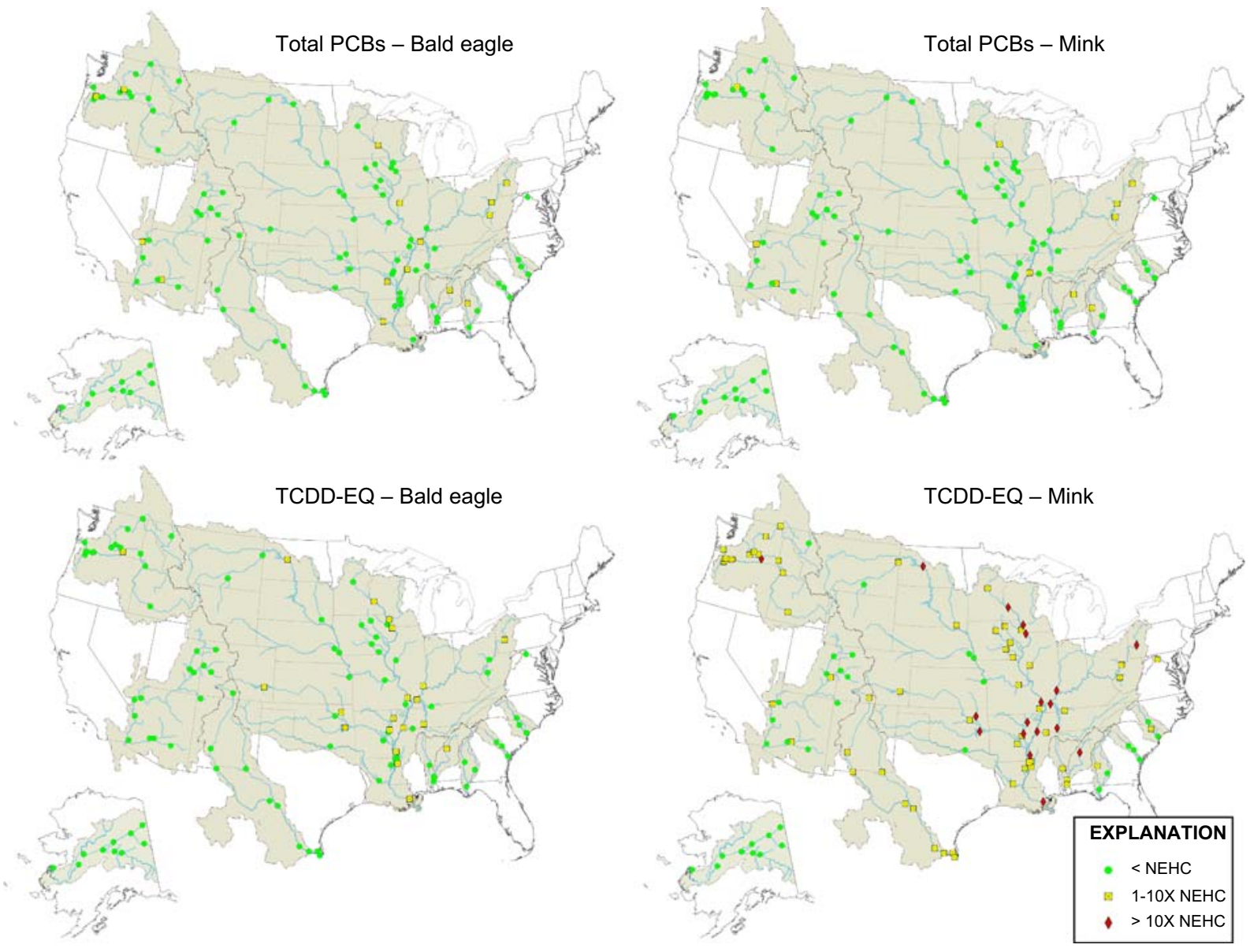

Fig. 3 (continued)

Environmental residues of DDT and its degradation products persist in many areas of historic use, primarily in agricultural areas and near manufacturing and formulation sites, even though the insecticide has been banned in the United States since 1972. Total DDT concentrations remained high in whole-body fish samples from multiple sampling sites in our study and represented a greater risk to bald eagle than mink. Total DDT concentrations exceeded the NEHC for bald eagle in at least one sample from all basins except the Yukon and Savannah River Basins, and the risk was greatest at sites in agricultural areas of the lower Columbia, Mississippi, Rio Grande and Colorado River Basins. Other studies have reported that DDT concentrations in these regions continue to represent a risk to avian wildlife (Grubb et al. 1990; Henny et al. 2004; Buck et al. 2005). Concentrations of $p, p^{\prime}$-DDE were associated with reproductive effects including eggshell thinning and lower productivity in osprey and bald eagle populations in the lower Columbia River Basin (Henny et al. 2004; Buck et al. 2005). Conversely, DDT and other contaminants did not affect bald eagle populations nesting along the Salt and Verde Rivers (Grubb et al. 1990), upstream of where our study indicated high risk of total DDT exposure to bald eagles in the Colorado River Basin. Other studies of bald eagles from our study area were not found in the scientific literature. Our screening level risk analysis indicated that bald eagles were at greatest risk to total DDT in winter nesting areas in the lower Mississippi River Basin (i.e., Arkansas and Mississippi). Mink were also at risk to total DDT exposure in the lower Mississippi River Valley at sites in watersheds farmed for cotton. We are not aware of specific studies evaluating total DDT 
Table 8 Toxicity reference value (TRV) for bald eagle and mink based on the no observed adverse effect level from dietary or tissue studies $^{\mathrm{a}}$, no effects hazard concentration $(\mathrm{NEHC})^{\mathrm{b}}$, and the number of samples and sites that exceed the $\mathrm{NEHC}^{\mathrm{c}}$

\begin{tabular}{|c|c|c|c|c|c|c|c|c|}
\hline \multirow[t]{2}{*}{ Contaminant } & \multicolumn{4}{|l|}{ Bald eagle } & \multicolumn{4}{|l|}{ Mink } \\
\hline & $\begin{array}{l}\text { TRV } \\
(\mathrm{mg} / \mathrm{kg} / \mathrm{d})\end{array}$ & $\begin{array}{l}\text { NEHC } \\
(\mu \mathrm{g} / \mathrm{g})\end{array}$ & $\begin{array}{l}\text { No. of } \\
\text { samples }\end{array}$ & $\begin{array}{l}\text { No. of } \\
\text { sites }\end{array}$ & $\begin{array}{l}\text { TRV } \\
(\mathrm{mg} / \mathrm{kg} / \mathrm{d})\end{array}$ & $\begin{array}{l}\text { NEHC } \\
(\mu g / g)\end{array}$ & $\begin{array}{l}\text { No. of } \\
\text { samples }\end{array}$ & $\begin{array}{l}\text { No. of } \\
\text { sites }\end{array}$ \\
\hline PentaCB & $\mathrm{N} / \mathrm{A}$ & $\mathrm{N} / \mathrm{A}$ & $\mathrm{N} / \mathrm{A}$ & $\mathrm{N} / \mathrm{A}$ & $7.25^{\mathrm{d}}$ & 53.3 & 0 & 0 \\
\hline $\mathrm{HCB}$ & $0.225^{\mathrm{e}}$ & 2.25 & 0 & 0 & $\mathrm{~N} / \mathrm{A}$ & $\mathrm{N} / \mathrm{A}$ & $\mathrm{N} / \mathrm{A}$ & N/A \\
\hline$\alpha-\mathrm{HCH}$ & $0.56^{\mathrm{f}}$ & 5.6 & 0 & 0 & $0.014^{\mathrm{f}}$ & 0.10 & 0 & 0 \\
\hline$\beta-\mathrm{HCH}$ & $0.56^{\mathrm{f}}$ & 5.6 & 0 & 0 & $0.31^{\mathrm{f}}$ & 2.28 & 0 & 0 \\
\hline$\gamma-\mathrm{HCH}$ & $2^{\mathrm{f}}$ & 20 & 0 & 0 & $6.15^{\mathrm{f}}$ & 45.2 & 0 & 0 \\
\hline$\delta-\mathrm{HCH}$ & $0.56^{\mathrm{f}}$ & 5.6 & 0 & 0 & $0.014^{\mathrm{f}}$ & 0.10 & 0 & 0 \\
\hline Aldrin & $\mathrm{N} / \mathrm{A}$ & N/A & $\mathrm{N} / \mathrm{A}$ & N/A & $0.154^{\mathrm{f}}$ & 1.13 & 0 & 0 \\
\hline Dieldrin & $0.077^{\mathrm{f}}$ & 0.77 & 0 & 0 & $0.015^{\mathrm{f}}$ & 0.11 & 6 & 4 \\
\hline Endrin & $0.010^{\mathrm{f}}$ & 0.10 & 5 & 2 & $0.038^{\mathrm{f}}$ & 0.28 & 2 & 1 \\
\hline ¿Chlordane & $2.14^{\mathrm{f}}$ & 21.4 & 0 & 0 & $1.9^{\mathrm{f}}$ & 14.0 & 0 & 0 \\
\hline इDDT & $6^{\mathrm{g}}$ & 0.27 & 92 & 36 & $0.62^{\mathrm{f}}$ & 4.56 & 5 & 4 \\
\hline Endosulfans & $10^{\mathrm{f}}$ & 100 & 0 & 0 & $0.12^{\mathrm{f}}$ & 0.88 & 0 & 0 \\
\hline $\mathrm{PCB}$ & $20^{\mathrm{g}}$ & 0.71 & 35 & 15 & $0.137^{\mathrm{f}}$ & 1.01 & 21 & 10 \\
\hline Methoxychlor & N/A & N/A & N/A & N/A & $3.1^{\mathrm{f}}$ & 22.8 & 0 & 0 \\
\hline Toxaphene & N/A & N/A & $\mathrm{N} / \mathrm{A}$ & $\mathrm{N} / \mathrm{A}$ & $6.2^{\mathrm{f}}$ & 45.6 & 0 & 0 \\
\hline TCDD-EQ & $0.000303^{\mathrm{g}}$ & 0.000016 & 31 & 20 & $0.00000027^{\mathrm{h}}$ & 0.0000019 & 185 & 76 \\
\hline Arsenic & $2.24^{\mathrm{i}}$ & 22.4 & 0 & 0 & $1.04^{\mathrm{i}}$ & 7.65 & 0 & 0 \\
\hline Cadmium & $1.47^{\mathrm{j}}$ & 14.7 & 0 & 0 & $0.77^{\mathrm{j}}$ & 5.66 & 0 & 0 \\
\hline Chromium & $2.66^{\mathrm{k}}$ & 26.6 & 4 & 3 & $2.40^{\mathrm{k}}$ & 17.7 & 7 & 5 \\
\hline Copper & $4.05^{1}$ & 40.5 & 0 & 0 & $5.60^{1}$ & 41.2 & 0 & 0 \\
\hline Mercury & $0.5^{\mathrm{m}}$ & 0.5 & 14 & 9 & $0.05^{\mathrm{n}}$ & 0.37 & 34 & 22 \\
\hline Nickel & $6.71^{\circ}$ & 67.1 & 0 & 0 & $1.70^{\circ}$ & 12.5 & 0 & 0 \\
\hline Lead & $1.63^{\mathrm{p}}$ & 16.3 & 0 & 0 & $4.7^{\mathrm{p}}$ & 34.6 & 0 & 0 \\
\hline Selenium & $0.4^{\mathrm{f}}$ & 4.0 & 3 & 1 & $0.154^{\mathrm{f}}$ & 1.13 & 51 & 19 \\
\hline Zinc & $14.49^{\mathrm{f}}$ & 145 & 1 & 1 & $123^{\mathrm{f}}$ & 904 & 0 & 0 \\
\hline
\end{tabular}

TRVs were not available for pentachloroanisole, Dacthal, and mirex for either bald eagle or mink. $\Sigma$ Chlordane is the sum of heptachlor epoxide, oxychlordane, cis-chlordane, trans-chlordane, cis-nonachlor, and trans-nonachlor, and $\Sigma$ DDT is the sum of $o, p^{\prime}$ and $p, p^{\prime}$-DDE, DDD, and DDT

$N / A$ Not available, penta $C B$ pentachlorobenzene, $H C B$ hexachlorobenzene

${ }^{a}$ Most TRVs were derived from adult dietary exposures. TRVs for EDDT, PCB, TCDD-EQ, and mercury in bald eagle were based on egg concentrations

${ }^{\mathrm{b}} \mathrm{NEHC}=$ dietary-based no adverse effects level TRV ( $\mathrm{mg} / \mathrm{kg}$ body weight/day)/food ingestion rate ( $\mathrm{kg}$ food/kg body weight/day). Food ingestion rate $=0.100 \mathrm{~kg}$ food $/ \mathrm{kg}$ body weight $/$ day for bald eagle and $0.136 \mathrm{~kg}$ food $/ \mathrm{kg}$ body weight/day for mink (USEPA 1993). NEHC=tissue-based no adverse effects level TRV ( $\mathrm{mg} / \mathrm{kg}$ egg)/biomagnification factor (BMF). The bald eagle BMFs were 28 for PCB, 19 for TCDD-EQ, 22 for $\mathrm{EDDT}$, and 1 for mercury (Giesy et al. 1995)

${ }^{\mathrm{c}}$ Number of samples $=409$ for all contaminants except for TCDD-EQ $(n=408)$ and pentachlorobenzene, aldrin, endosulfans, and methoxychlor $(n=135)$. Number of sites $=111$ except for pentachlorobenzene, aldrin, endosulfans, and methoxychlor $(n=37)$

${ }^{\mathrm{d}}$ Linder et al. 1980

${ }^{\mathrm{e}}$ Hill and Camardese 1986

${ }^{\mathrm{f}}$ Sample et al. 1996

${ }^{\mathrm{g}}$ Elliott and Harris 2001

${ }^{\mathrm{h}}$ Tillitt et al. 1996

${ }^{\mathrm{i}}$ USEPA 2005a

${ }^{\mathrm{j}}$ USEPA 2005b

${ }^{\mathrm{k}}$ USEPA $2005 \mathrm{c}$

${ }^{1}$ USEPA 2007a

${ }^{\mathrm{m}}$ Giesy et al. 1995

${ }^{\mathrm{n}}$ USEPA 1995

${ }^{\circ}$ USEPA $2007 \mathrm{~b}$

${ }^{\mathrm{p}}$ USEPA 2005d 


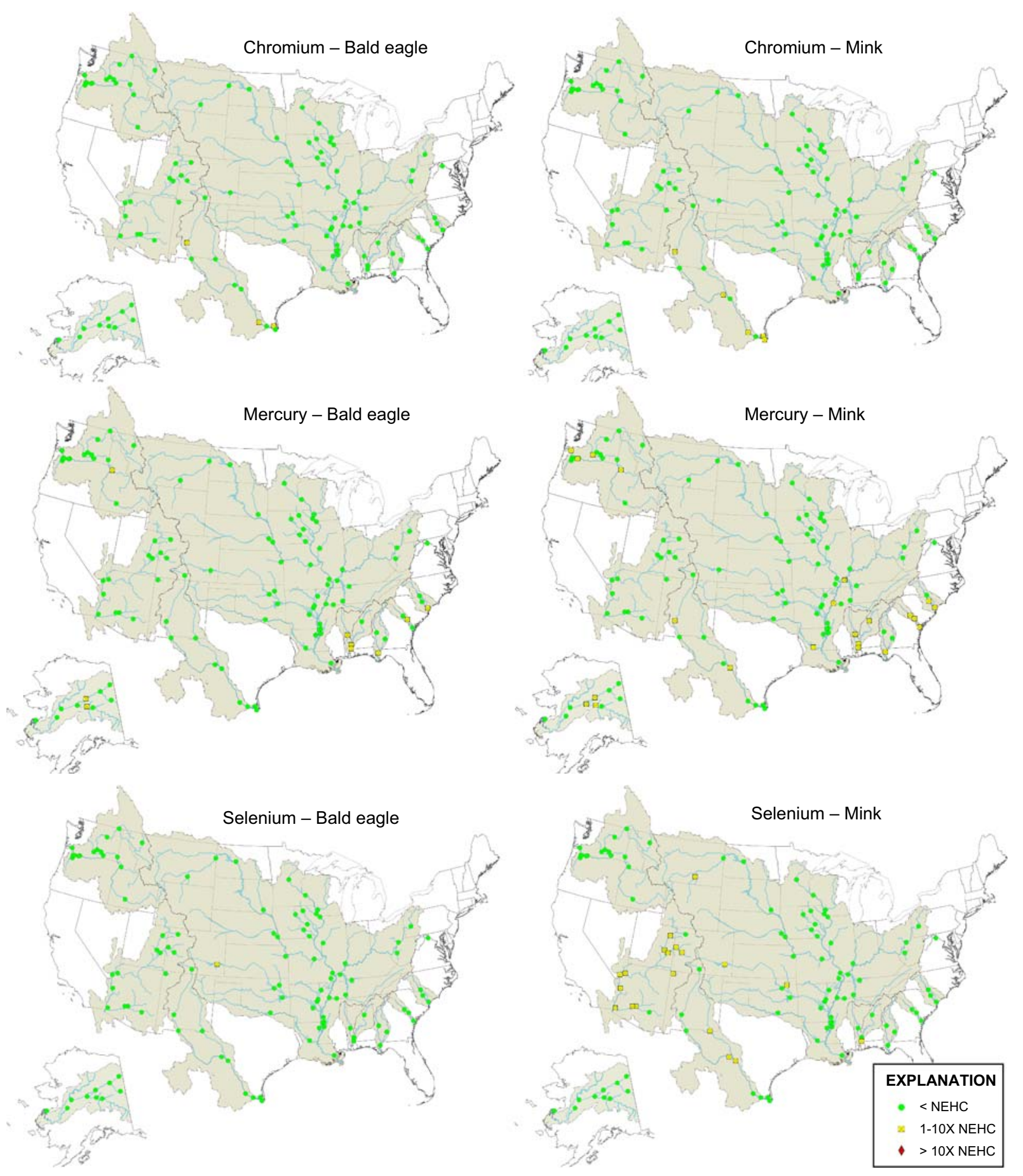

Fig. 4 Sites that exceed the no effects hazard concentration (NEHC) for selected elemental contaminants. See Fig. 1 for specific site locations and Table 8 for NEHC concentrations 
concentrations in piscivorous mammal populations from this region.

PCBs were used historically as dielectric, hydraulic, and heat transfer fluids; lubricants; and in carbonless copy paper until the U.S. ban in 1979. Total PCB concentrations exceeded NEHCs for bald eagle and mink at multiple sites. Mink are among the most sensitive organisms to PCBs with their survival and reproduction affected by small dietary PCB doses (Leonards et al. 1995). Risk associated with total PCBs to mink generally was greatest in industrialized watersheds and near historical production and formulation facilities. NEHCs for both mink and bald eagle were exceeded at Sites 23, 24, 67, 76, 111, 320, 324, 327, and 330. Elevated PCB concentrations have been previously documented in water and biota near Site 327 (USFWS 1996; Schmitt et al. 1999; Zappia 2002), and PCBs were identified as a potential cause of reproductive dysfunction in mink from the coastal plain of Georgia, South Carolina, and North Carolina (Osowski et al. 1995). We are not aware of contaminant studies evaluating the risk of total PCBs to piscivorous wildlife in the Mississippi River Basin downstream from Memphis (Site 76) or the Ohio River Valley (Sites 23, 24, and 67). Mink typically do not occur in the southwestern United States, but other piscivorous mammals [i.e., raccoon (Procyon lotor)] may be at risk to total PCBs near Sites 320 and 324. The Gila River at Site 324 has low or no flow for part of the year and is recharged by wastewater treatment plant effluent and urban runoff from the Phoenix area. The Gila River supports contaminated prey items including fish with elevated concentrations of organochlorine pesticides and total PCBs (Hinck et al. 2007). Therefore, contaminant exposure to piscivorous wildlife may be increased by limited water and food resources in this arid region, where riparian wildlife concentrate. Moreover, bald eagles breeding in southern Arizona habitually forage in the same areas of free-flowing water, where fish are the most commonly consumed prey item (Haywood and Ohmart 1986; Grubb 1995).

PCBs were formulated as complex mixtures comprising as many as 209 structurally similar compounds (congeners) that vary widely in toxicity and persistence. The toxicity of PCBs to mink, bald eagles, and other organisms is largely attributable to relatively few congeners, specifically the highly toxic non-ortho- and mono-ortho-chloro substituted com- pounds that can assume a planar molecular configuration (Aulerich and Ringer 1977; Peterson et al. 1993; Tillitt et al. 1996). These compounds are structurally similar to 2,3,7,8-tetrachloro- $p$-dioxin (TCDD) and are among the compounds collectively known as planar halogenated hydrocarbons (PHHs). Concentrations of PHHs in fish can vary over orders of magnitude depending upon the original composition of the mixture released to the environment, weathering, and toxicokinetic factors, and they largely determine the risk assessment of PCBs in the environment (Safe 1990; van den Berg et al. 2006). It has been widely accepted that concentrations of individual congeners, specifically the planar congeners, rather than total PCB concentrations are necessary to assess the risk of PCBs to piscivorous wildlife. However, recent studies indicate that total PCBs measurements can be used to assess the toxicological risks associated with dioxin-like PCB concentrations in whole-body benthivorous and piscivorous fish (Bhavsar et al. 2007a, b). High-resolution analyses required for PHHs were beyond the scope of our studies; therefore, we determined dioxin-like activity (i.e., TCDD-EQ) with the H4IIE bioassay. As measured in our samples, TCDD-EQ includes the contributions of all the PHHs including the planar PCB congeners. Dioxin-like activity (as TCDD-EQ) was elevated at many of the sites where total PCB concentrations were also comparatively high, indicating that much of the dioxin-like activity was attributable to PCBs. However, the overall risk of TCDD-EQ to bald eagle and mink was more widespread than risk of PCBs, indicating some contributions from other PHHs at sites with high TCDD-EQ concentrations but low total PCB concentrations. TCDD-EQ-related risk was greatest in the Columbia, Rio Grande, Mobile, and Mississippi River Basins, but was minimal in the Yukon, Colorado, Apalachicola, Savannah, and Pee Dee River Basins.

The risk of mercury to piscivorous wildlife was greatest at sites in the southeastern United States (Mobile, Apalachicola, Savannah, and Pee Dee River Basins) and Yukon River Basin. Sources of $\mathrm{Hg}$ in these basins include historical gold and cinnabar mines, releases from chemical manufacturing and coal-fired power plants, atmospheric deposition, and wetlands (Rudd 1995; Brumbaugh et al. 2001; Eisler 2004; Paller et al. 2004; Warner et al. 2005). Orihel et al. (2007) demonstrated that organic mercury deposited 
directly to aquatic ecosystem was readily converted to methylmercury, which is available to biota. This relationship may explain the high fish concentrations and associated risk of mercury in the southeastern United States and Yukon River Basin, where mercury methylation rates are known to be high (Brumbaugh et al. 2001). Other studies also have reported that mercury concentrations in these regions represent a risk to avian and mammalian wildlife (Halbrook et al. 1994; Osowski et al. 1995; Ambrose et al. 2000; Adair et al. 2003). Mercury has been suspected to cause reproductive effects, organ toxicity, and mortality in prothonotary warbler (Protonotaria citrea), mink, and river otter throughout the southeastern United States (Halbrook et al. 1994; Osowski et al. 1995; Adair et al. 2003). Ambrose et al. (2000) reported that mercury concentrations in peregrine falcon (Falco peregrinus anatum) breeding in the Yukon River Basin were associated with lower nest success. Conversely, mercury concentrations in bald eagle eggs from the lower Columbia River Basin were not associated with lower productivity (Anthony et al. 1993; Buck et al. 2005).

Sources of excess selenium include natural weathering of seleniferous shales, irrigation practices, uranium ore and coal extraction, and coal-fired power plants (Sorenson 1991). The risk of selenium to bald eagle was lower than to mink. Among basins, mink in the Colorado River Basin were at greatest risk to selenium exposure. Irrigation and mining throughout these areas accelerate the rates of the processes controlling the release and distribution of this naturally occurring element. Selenium exposure to wildlife is most commonly associated with toxicity to birds (Lemly 1996; Hamilton 2004). Historically, avian wildlife was impacted by selenium contamination in the Colorado River Basin near Site 312 (Stephens et al. 1992), but we are not aware of studies evaluating selenium toxicity to piscivorous mammals in this basin.

The wildlife risk analysis presented here is a screening level or Tier 1 assessment. As such, it was based on the most conservative (i.e., lowest) TRVs available to identify all potential contaminants of concern (USEPA 1998). The conservative TRVs we used included values extrapolated from receptors other than bald eagle and mink. As noted by Sample and Suter (1999), such an approach indicates only whether harmful effects are possible, not whether they are probable. Consequently, the use of the most conservative TRVs may overestimate the actual risk of some contaminants to wildlife. For example, we initially used a TRV for mercury in bald eagle $(0.0064 \mathrm{mg} / \mathrm{kg} / \mathrm{d})$ derived from a multi-generational study of mallards (Anas platyrychos; Heinz 1979; Sample et al. 1996). Based on this TRV, Yeardley et al. (1998) estimated that mercury concentrations in fish from $98 \%$ of the lakes in the northeastern United States represented a risk to piscivorous birds. Our NEHC calculated from this TRV $(0.064 \mathrm{~g} / \mathrm{g})$ similarly indicated that bald eagles were at risk from mercury at most of our sites (105 of 109 sites), which might be interpreted as indicative of widespread mercury poisoning of bald eagles in the conterminous United States. This conclusions would contradict current data indicating the recovery of U.S. bald eagle populations and their recent removal from the Endangered Species List (U.S. Fish and Wildlife Service 2007), and would indicate that an alternative TRV should be considered. Therefore, we selected a NEHC of $0.5 \mu \mathrm{g} / \mathrm{g}$ developed specifically to estimate mercury risk to bald eagles (Giesy et al. 1995), which resulted in considerably lower estimates of risk to bald eagles at our sites; only nine sites had mercury concentrations $>0.5 \mu \mathrm{g} / \mathrm{g}$. However, there is evidence that piscivorous birds are being negatively affected by mercury in fish elsewhere (e.g., Scheuhammer and Blancher 1994; Scheuhammer et al. 1998; Nocera and Taylor 1998). One might therefore argue that bald eagle populations would be increasing more rapidly if not for mercury, which would support the use of the lower TRVs. This example highlights the limitations of conservative TRVs and the importance of qualifying their use in the screening level phase of wildlife risk analysis. Regardless of the TRV employed, sites at risk would need to be evaluated further to fully characterize the exposure and ecological effects of specific contaminants to bald eagles or mink (e.g., Tier 2 risk assessment).

There are other assumptions and uncertainties associated with the approach we used to evaluate risk to piscivorous wildlife. Some of the NOAELs used here were derived from studies conducted several decades ago, and other NOAELs have been proposed and used elsewhere. Consensus NOAELs based on whole-body fish concentrations, such as those adopted for arsenic, cadmium, chromium, copper, nickel, and lead, are not available for many contaminants. The use of other NOAELs would result in different 
NEHCs and could change the perceived risk to piscivorous wildlife. Other wildlife food chain analyses incorporate water and sediment/soil concentrations to estimate contaminant concentrations in fish and evaluate the risk of contaminants to piscivorous wildlife because fish concentrations are not available. While more recent toxicity thresholds may be available for water, soil, and sediment, there are additional uncertainties associated with estimating exposure to piscivorous wildlife from these endpoints. Factors such as organic carbon content, sulfides, hardness, and $\mathrm{pH}$ can influence the bioavailability and toxicity of contaminants (e.g., Björnberg et al. 1988; Chapman et al. 1998; Heekyoung Choi et al. 1998). These points emphasize that updated consensus toxicity thresholds based on whole-body fish concentrations associated with reproductive performance, growth, and survival are needed, especially for contaminants such as pentachloroanisole, Dacthal, and mirex for which toxicity thresholds are not currently available. However, studies determining toxicity thresholds in whole-body fish are not likely to be conducted for some of these contaminants because of time and expense requirements for multi-generational studies with birds and mammals and the current focus of research on new or emerging contaminants.

The selection of appropriate receptors in the analysis is important. Bald eagles and mink consume large, adult fish such as those collected and analyzed in this study and are therefore reasonable receptors. The risk of contaminants to other piscivorous wildlife such as belted kingfisher (Ceryle alcyon), Caspian tern (Sterna caspia), or common loons (Gavia immer) may be overestimated if these species are used in the model, because they consume smaller fish that are presumed to have lower contaminant concentrations than larger fish because of their shorter lifespans, higher growth rates, and higher metabolism (Scheuhammer et al. 1998; Rose et al. 1999). It is also unlikely that the diet of adult bald eagles or mink would be entirely composed of fish from one site, which is tacitly assumed in this approach. Nevertheless, this assumption may be valid for nestling birds or cubs fed locally procured prey items. Moreover, the risk of accumulative contaminants to nestlings/cubs and juveniles may be greater because of their small body size and correspondingly higher food ingestion rates compared to adult wildlife. Inexperienced juveniles also may have greater exposure to chemical contaminants because of their initial small home range or when they scavenge on dead or dying fish associated with natural or anthropogenic events.

As a final consideration, this assessment assumes that the contaminants act independently. Many of the contaminants co-occur at the sites, and their effects are not necessarily independent or additive (e.g., Wren et al. 1987). Effects also may vary depending on other factors such as the disease, nutritional, and reproductive status of the organism. Nevertheless, the NOAELs and subsequent NEHCs represent conservative, screening-level criteria for the assessment of potential adverse effects in wildlife from chemical contaminants in the fish that they consume.

Overall, concentrations of many persistent, bioaccumulative organochlorine chemical residues have declined over the past two decades. Our results indicate that organochlorine residues, in particular $p, p^{\prime}$-DDE and total PCBs, remain a risk to piscivorous wildlife at some sites. Concentrations of mercury and selenium, which have not declined, also represent a risk at a number of sites. In addition, mercury and selenium concentrations in aquatic systems may rise because of increasing global coal combustion and subsequent atmospheric deposition (USEPA 1997; Mason et al. 1999). The full geographic extent of the risk associated with contaminants in fish cannot be determined from our data because some of the sites were not selected in a truly unbiased manner and only screening level analyses were conducted. Regardless, our findings indicate that environmental contaminants are a risk to piscivorous wildlife at some sites. Characterization of exposure and ecological effects for contaminants at specific sites would be the next step in refining the risks determined in our screening level wildlife risk analysis.

Acknowledgements This study was conducted as a component of the Large River Monitoring Network of the Biomonitoring Environmental Status and Trends (BEST) Project. This project is managed by the Status and Trends Program of the U.S. Geological Survey. A. Bernhardt, N. Beyer, and G. Linder reviewed earlier versions of this paper and provided additional information.

\section{References}

Adair, B. M., Reynolds, K. D., McMurry, S. T., \& Cobb, G. P. (2003). Mercury occurrence in prothonotary warblers (Protonotaria citrea) inhabiting a national priority list site and reference areas in southern Alabama. Archives of Environmental Contamination and Toxicology, 44, 265-271. 
Ambrose, R. E., Matz, A., Swem, T., \& Bente, P. (2000). Environmental contaminants in American and arctic peregrine falcon eggs in Alaska, 1979-95. NAES-TR00-02, US Fish and Wildlife Service, Fairbanks, Alaska.

Anderson, D. W., Jehl Jr., J. R., Risebrough, R. W., Woods Jr., L. A., DeWeese, L. R., \& Edgecomb, W. G. (1975). Brown pelicans: improved reproduction off the southern California coast. Science, 190, 806-808.

Ankley, G. T., Tillitt, D. E., Giesy, J. P., Jones, P. D., \& Verbrugge, D. A. (1991). Bioassay-derived 2,3,7,8-tetrachlorodibenzo- $p$-dioxin equivalents in PCB-containing extracts from the flesh and eggs of Lake Michigan chinook salmon (Oncorhynchus tshawytscha) and possible implications for reproduction. Canadian Journal of Fisheries and Aquatic Sciences, 48, 1685-1690.

Anthony, R. G., Garrett, M. G., \& Schuler, C. A. (1993). Environmental contaminants in bald eagles in the Columbia River estuary. Journal of Wildlife Management, 57, 10-19.

Aulerich, R. J., \& Ringer, R. K. (1977). Current status of PCB toxicity to mink, and effect on their reproduction. Archives of Environmental Contamination and Toxicology, 6, 279-292.

Barr, J. F. (1986). Population dynamics of the common loon (Gavia immer) associated with mercury-contaminated waters in northwestern Ontario. Canadian Wildlife Service Occasional Paper 56.

Baumann, P. C., \& Gillespie, R. B. (1986). Selenium bioaccumulation in gonads of largemouth bass and bluegill from three power plant cooling reservoirs. Environmental Toxicology and Chemistry, 5, 695-701.

Beckvar, N., Dillion, T. M., \& Read, L. B. (2005). Approaches for linking whole-body fish tissue residues of mercury and DDT to biological effects thresholds. Environmental Toxicology and Chemistry, 24, 2094-2105.

Blus, L. J. (1996). DDT, DDD, and DDE in birds. In W. N. Beyer, G. H. Heinz, \& A. W. Redmon-Norwood (Eds.), Environmental contaminants in wildlife: Interpreting tissue concentrations (pp. 49-71). Boca Raton, Florida: Lewis Publishers.

Björnberg, A., Hakanson, L., \& Lundbergh, K. (1988). A theory on the mechanisms regulating the bioavailability of mercury in natural waters. Environmental Pollution, 49, 53-61.

Brumbaugh, W. G., Krabbenhoft, D. P., Helsel, D. R., Wiener, J. G., \& Echols, K. R. (2001). A national pilot study of mercury contamination of aquatic ecosystems along multiple gradients: bioaccumulation in fish. Biological Science Report, 2001-0009, US Geological Survey, Columbia, Missouri.

Bhavsar, S. P., Fletcher, R., Hayton, A., Reiner, E. J., \& Jacoson, D. A. (2007a). Composition of dioxin-like PCBs in fish: An application for risk assessment. Environmental Science and Technology, 41, 3096-3102.

Bhavsar, S. P., Hayton, A., Reiner, E. J., \& Jackson, D. A. (2007b). Estimating dioxin-like polychlorinated biphenyl toxic equivalents from total polychlorinated biphenyl measurements in fish. Environmental Toxicology and Chemistry, 26, 1622-1628.

Buck, J. A., Anthony, R. G., Schular, C. A., Isaacs, F. B., \& Tillitt, D. E. (2005). Changes in productivity and contaminants in bald eagles nesting along the lower Columbia River, USA. Environmental Toxicology and Chemistry, 24, 1779-1792.
Chapman, P. M., Wang, F., Janssen, C., Persoonne, J., \& Allen, H. E. (1998). Ecotoxicology of metals in aquatic sediments: binding and release, bioavailability, risk assessment, and remediation. Canadian Journal of Fisheries and Aquatic Sciences, 55, 2221-2243.

Eisler, R. (1985). Cadmium hazards to fish, wildlife, and invertebrates: A synoptic review. Biological report, 85 (1.2), US Fish and Wildlife Service, Washington, D.C.

Eisler, R. (1986). Chromium hazards to fish, wildlife, and invertebrates: A synoptic review. Biological report, 85 (1.6), US Fish and Wildlife Service, Washington, D.C.

Eisler, R. (1990). Chlordane hazards to fish, wildlife, and invertebrates: A synoptic review. Biological report, 85 (1.21), US Fish and Wildlife Service, Washington, D.C.

Eisler, R. (2004). Mercury hazards from gold mining to humans, plants, and animals. Review of Environmental Contamination and Toxicology, 181, 139-198.

Elliott, J. E., \& Harris, M. L. (2001). An ecotoxicological assessment of chlorinated hydrocarbon effects on bald eagle populations. Reviews in Toxicology, 4, 1-60.

Giesy, J. P., Bowerman, W. W., Mora, M. A., Verbrugge, D. A., Othoudt, R. A., Newsed, J. L., et al. (1995). Contaminants in fishes from Great Lakes-influenced sections and above dams of three Michigan rivers: III. Implications for health of bald eagles. Archives of Environmental Contamination and Toxicology, 29, 309-321.

Gilderhus, P. A. (1966). Some effects of sublethal concentrations of sodium arsenate on bluegills and the aquatic environment. Transactions of the American Fisheries Society, 95, 289-296.

Grubb, T. G. (1995). Food habits of bald eagles breeding in the Arizona desert. Wilson Bulletin, 107, 258-274.

Grubb, T. G., Wiemeyer, S. N., \& Kiff, L. F. (1990). Eggshell thinning and contaminant levels in bald eagle eggs from Arizona, 1977 to 1985. Southwest Naturalist, 35, 298301.

Halbrook, R. S., Jenkins, J. H., Bush, P. B., \& Seabolt, N. D. (1994). Sublethal concentrations of mercury in river otters: Monitoring environmental contamination. Archives of Environmental Contamination and Toxicology, 27, 306-310.

Hamilton, S. J. (2004). Review of selenium toxicity in the aquatic food chain. Science of the Total Environment, 326, $1-31$.

Hansen, J. A., Welsh, P. G., Lipton, J., \& Suedkamp, M. J. (2002). The effects of long-term cadmium exposure on the growth and survival of juvenile bull trout (Salvelinus confluentus). Aquatic Toxicology, 58, 165-174.

Haywood, D. D., \& Ohmart, R. D. (1986). Utilization of benthic-feeding fish by inland breeding bald eagles. Condor, 88, 35-42.

Heaton, S. N., Bursian, S. J., Giesy, J. P., Tillitt, D. E., Render, J. A., Jones, P. D., et al. (1995). Dietary exposure of mink to carp from Saginaw Bay, Michigan: 1. Effects on reproduction and survival, and potential risks to wild mink populations. Archives of Environmental Contamination and Toxicology, 28, 334-343.

Heekyoung Choi, M., Cehc Jr., J. J., \& Lagunas-Solar, M. C. (1998). Bioavailability of methylmercury to Sacramento blackfish (Orthodon microlepidotus): Dissolved organic carbon effects. Environmental Toxicology and Chemistry, 17, 695-701. 
Heinz, G. H. (1979). Methyl mercury: reproductive and behavioral effects on three generations of mallard ducks. Journal of Wildlife Management, 43, 394-401.

Henny, C. J., Grove, R. A., Kaiser, J. L., \& Bentley, V. R. (2004). An evaluation of osprey eggs to determine spatial residue patterns and effects of contaminants along the lower Columbia River, U.S.A. In R. D. Chancellor, \& B. U. Meyburg (Eds.), Raptors Worldwide (pp. 369-388). Berlin, Germany: Hancock House Publishers.

Hill, E. F., \& Camardese, M. B. (1986). Lethal dietary toxicities of environmental contaminants and pesticides to coturnix. Technical report 2, US Fish and Wildlife Service, Washington D.C.

Hinck, J. E., Blazer, V. S., Denslow, N. D., Echols, K. R., Gale R. W., Wieser, C., et al. (2008). Chemical contaminants, health indicators, and reproductive biomarker responses in fish from rivers in the Southeastern U.S. Science of the Total Environment, 390(2-3), 538-557.

Hinck, J. E., Blazer, V. S., Denslow, N. D., Echols, K. R., Gross, T. S., May, T. W., et al. (2007). Chemical contaminants, health indicators, and reproductive biomarker responses in fish from the Colorado River and its tributaries. Science of the Total Environment, 378, 376-402.

Hinck, J. E., Schmitt, C. J., Blazer, V. S., Denslow, N. D., Bartish, T. M., Anderson, P. J., et al. (2006a). Environmental contaminants and biomarker responses in fish from the Columbia River and its tributaries: Spatial and temporal trends. Science of the Total Environment, 366, 549-578.

Hinck, J. E., Schmitt, C. J., Echols, K. R., May, T. W., Orazio, C. E., \& Tillitt, D. E. (2006b). Environmental contaminants in fish and their associated risk to piscivorous wildlife in the Yukon River Basin, Alaska. Archives of Environmental Contamination and Toxicology, 51, 661-672.

Holcombe, G. W., Benoit, D. A., Leonard, E. N., \& McKim, J. M. (1976). Long-term effects of lead exposure on three generations of brook trout (Salvelinus fontinalis). Journal of Fisheries Research Board of Canada, 33, 1731-1741.

Hornshaw, T. C., Aulerich, R. J., \& Johnson, H. E. (1983). Feeding great lakes fish to mink: effects on mink and accumulation and elimination of PCBs by mink. Journal of Toxicology and Environmental Health, 11, 933-946.

Keith, L. H. (1991). Environmental Sampling and Analysis. (Boca Raton, Florida: Lewis Publishers).

Lemly, A. D. (1996). Selenium in aquatic organisms. In W. N. Beyer, G. H. Heinz, \& A. W. Redmon-Norwood (Eds.), Environmental contaminants in wildlife: Interpreting tissue concentrations (pp. 427-445). Boca Raton, Florida: Lewis Publishers.

Leonards, P. E. G., de Vries, T. H., Minnaard, W., Stuijfzand, S., de Voogt, P., Cofino, W. P., et al. (1995). Assessment of experimental data on PCB-induced reproduction inhibition in mink, based on an isomer- and congener-specific approach using 2,3,7,8-tetrachlorodibenzo-p-dioxin toxic equivalency. Environmental Toxicology and Chemistry, 14, 639-652.

Linder, R., Scotti, T., Goldstein, J., McElroy, K., \& Walsh, D. (1980). Acute and subchronic toxicity of pentachlorobenzene. Journal of Environmental Pathology and Toxicology, 4, 183-196.

Mason, R. P., Laporte, J. M., \& Andres, S. (1999). Factors controlling the bioaccumulation of mercury, methylmer- cury, arsenic, selenium, and cadmium by freshwater invertebrates and fish. Archives of Environmental Contamination and Toxicology, 38, 283-297.

Mayer, F. L., Jr., Mehrle, P. M., Jr., \& Dwyer, W. P. (1975). Toxaphene effects on reproduction, growth, and mortality of brook trout. Report EPA-600/3-75/013, US Environmental Protection Agency, Duluth, Minnesota.

McGreachy, S. M., \& Dixon, D. G. (1992). Whole-body arsenic concentrations in rainbow trout during acute exposure to arsenate. Ecotoxicology and Environmental Safety, 24, 301-308.

Newell, A. J., Johnson, D. W., \& Allen, L. K. (1987). Niagara River biota contamination project: fish flesh criteria for piscivorous wildlife. Bureau of Environmental Protection Technical Report 87-3, New York State Department of Environmental Conservation, Buffalo, New York.

Nocera, J. J., \& Taylor, P. D. (1998). In situ behavioral responses of common loons associated with elevated mercury $(\mathrm{Hg})$ exposure. Conservation Ecology, 2, 10.

Nosek, J. A., Craven, S. R., Sullivan, J. R., Hurley, S. S., \& Peterson, R. E. (1992). Toxicity and reproductive effects of 2,3,7,8-tetrachlorodibenzo-p-dioxin in ring-necked pheasant hens. Journal of Toxicology and Environmental Health, 35, 187-198.

Orihel, D., Paterson, M. J., Blanchfield, P. J., Bodaly, R. J., \& Hintelmann, H. (2007). Experimental evidence of a linear relationship between inorganic mercury loading and methylmercury accumulation in aquatic biota. Environmental Science and Technology, 41, 4952-4958.

Osowski, S. L., Brewer, L. W., Baker, O. E., \& Cobb, G. P. (1995). The decline of mink in Georgia, North Carolina, and South Carolina: the role of contaminants. Archives of Environmental Contamination and Toxicology, 29, 418-423.

Paller, M. H., Bowers, J. A., Littrell, J. W., \& Guanlao, A. V. (2004). Influences on mercury bioaccumulation factors for the Savannah River. Archives of Environmental Contamination and Toxicology, 46, 236-243.

Peakall, D. B. (1996). Dieldrin and other cyclodiene pesticides in wildlife. In W. N. Beyer, G. H. Heinz, \& A. W. Redmon-Norwood (Eds.), Environmental contaminants in wildlife: Interpreting tissue concentrations (pp. 73-98). Lewis Publishers: Boca Raton, Florida.

Peterson, R. E., Theobold, H. M., \& Kimmel, G. L. (1993). Developmental and reproductive toxicity of dioxins and related compounds: Cross species comparisons. Critical Reviews in Toxicology, 23, 283-335.

Reichel, W. L., Schmeling, S. K., Cromartie, E., Kaiser, T. E., Krynitsky, A. J., Lamont, T. G., et al. (1984). Pesticide, PCB, and lead residues and necropsy data for bald eagles from 32 states 1978-1981. Environmental Monitoring and Assessment, 4, 395-403.

Rose, J., Hutcheson, M. S., Rowan West, C., Pancorbo, O., Hulme, K., Cooperman, A., et al. (1999). Fish mercury distribution in Massachusetts, USA Lakes. Environmental Toxicology and Chemistry, 18, 1370-1379.

Rudd, J. W. (1995). Sources of mercury to freshwater ecosystems: A review. Water Air and Soil Pollution, 80, 697-713.

Safe, S. (1990). Polychlorinated biphenyls (PCBs), dibenzo-pdioxins (PCDDs), dibenzofurans (PCDFs), and related compounds: environmental and mechanistic considera- 
tions which support the development of toxic equivalency factors (TEFs). Critical Reviews in Toxicology, 21, 51-88.

Sample, B. E., \& Suter, G. W. (1999). Ecological risk assessment in a large river-reservoir: 4. piscivorous wildlife. Environmental Toxicology and Chemistry, 18, 610-620.

Sample, B. E., Opresko, D. M., \& Suter, G. W. (1996). Toxicological benchmarks for wildlife: 1996 revision. Report ES/ER/TM-86/R3, Oak Ridge National Laboratory, Oak Ridge, Tennessee.

Scheuhammer, A. M., Atchison, C. M., Wong, A. H. K., \& Evers, D. C. (1998). Mercury exposure in breeding Common Loons (Gavia immer) in central Ontario, Canada. Environmental Toxicology and Chemistry, 17, 191-196.

Scheuhammer, A. M., \& Blancher, P. J. (1994). Potential risk to Common Loons (Gavia immer) from methylmercury exposure in acidified lakes. Hydrobiologia, 279/280, 445-455.

Schmitt, C. J. (2002). Organochlorine chemical residues in fish from the Mississippi River Basin, 1995. Archives of Environmental Contamination and Toxicology, 43, 81-97.

Schmitt, C. J. (2004). Concentrations of arsenic, cadmium, copper, lead, selenium, and zinc in fish from the Mississippi River Basin, 1995. Environmental Monitoring and Assessment, 90, 289-321.

Schmitt, C. J., Brumbaugh, W. G., Linder, G. L., \& Hinck, J. E. (2006). A screening-level assessment of lead, cadmium, and zinc in fish and crayfish from northeastern Oklahoma, USA. Environmental Geochemistry and Health, 28, 445-471.

Schmitt, C. J., Hinck, J. E., Blazer, V. S., Denslow, N. D., Dethloff, G. M., Bartish, T. M., et al. (2005). Environmental contaminants and biomarker responses in fish from the Rio Grande and its U.S. tributaries: Spatial and temporal trends. Science of the Total Environment, 350, 161-193.

Schmitt, C. J., Zajicek, J. L., May, T. W., \& Cowman, D. F. (1999). National contaminant biomonitoring program: Concentrations of organochlorine chemical residues and elemental contaminants in U.S. freshwater fish, 19761986. Reviews of Environmental Contamination and Toxicology, 162, 43-104.

Shubat, P. J., \& Curtis, L. R. (1986). Ration and toxicant preexposure influence dieldrin accumulation by rainbow trout (Salmo gairdneri). Environmental Toxicology and Chemistry, 5, 69-77.

Sorenson, E. (1991). Metal poisoning in fish. (Boca Raton, Florida: CRC Press).

Spehar, R. L. (1976). Cadmium and zinc toxicity to flagfish (Jordanella floridae). Journal of Fisheries Research Board of Canada, 33, 1939-1945.

Stephens, D. W., Waddell, B., Peltz, L. A., \& Miller, J. B. (1992). Detailed study of selenium and selected elements in water, bottom sediment, and biota associated with irrigation drainage in the middle Green River basin, Utah, 1988-90. Water Resources Investigations Report, 924084, US Geological Survey, Denver, Colorado.

Stouthart, X. J. H. X., Haans, J. L. M., Lock, R. A. C., \& Wendelaar-Bonga, S. E. (1996). Effects of water $\mathrm{pH}$ on copper toxicity to early life stages of the common carp (Cyprinus carpio). Environmental Toxicology and Chemistry, 15, 376-383.

Tillitt, D. E., Gale, R. W., Meadows, J. C., Zajicek, J. L., Peterman, P. H., Heaton, S. N., et al. (1996). Dietary exposure of mink to carp from Saginaw Bay 3, characterization of dietary exposure to planar halogenated hydrocarbons, dioxin equivalents, and biomagnifications. Environmental Science and Technology, 30, 283-291.

Tillitt, D. E., Giesy, J. P., \& Ankley, G. T. (1991). Characterization of the H4IIE rat hepatic cell bioassay as a tool for assessing toxic potency of planar halogenated hydrocarbons in environmental samples. Environmental Science and Technology, 25, 87-92.

U.S. Environmental Protection Agency (1993). Wildlife exposure factors handbook. EPA/600/R093/187, Office of Health and Environmental Assessment and Office of Research and Development, Washington, D.C.

U.S. Environmental Protection Agency (1995). Great lakes water quality initiative criteria documents for the protection of wildlife. EPA-820\b-95\008, Office of Water, Washington, D.C.

U.S. Environmental Protection Agency (1997). Mercury study report to Congress. EPA-452/R-97-003, Office of Research and Development, Washington, D.C.

U.S. Environmental Protection Agency (1998). Guidelines for ecological risk assessment. EPA/630/R-95/002F, Washington, D.C.

U.S. Environmental Protection Agency (2005a). Ecological soil screening levels for arsenic. Interim Final OSWER Directive 9285.7-62, Office of solid waste and emergency response, Washington, D.C.

U.S. Environmental Protection Agency (2005b). Ecological soil screening levels for cadmium. Interim Final OSWER Directive 9285.7-65, Office of Solid Waste and Emergency Response, Washington, D.C.

U.S. Environmental Protection Agency (2005c). Ecological soil screening levels for chromium. Interim final OSWER Directive 9285.7-66, Office of Solid Waste and Emergency Response, Washington, D.C.

U.S. Environmental Protection Agency (2005d). Ecological soil screening levels for lead. Interim final OSWER Directive 9285.7-70, Office of Solid Waste and Emergency Response, Washington, D.C.

U.S. Environmental Protection Agency (2007a). Ecological soil screening levels for copper. Interim final OSWER Directive 9285.7-68, Office of Solid Waste and Emergency Response, Washington, D.C.

U.S. Environmental Protection Agency (2007b). Ecological soil screening levels for nickel. Interim final OSWER Directive 9285.7-76, Office of Solid Waste and Emergency Response, Washington, D.C.

U.S. Fish and Wildlife Service (1996). An investigation into the uptake of contaminants in largemouth bass and sediment from the lower Tombigbee and Mobile Rivers. Ecological Services Office Report 06-196-03-00-A, Daphne, Alabama.

U.S. Fish and Wildlife Service (2007). Endangered and threatened wildlife and plants; Removing the bald eagle in the lower 48 states from the list of endangered and threatened wildlife, 50 CFR Part 17. Federal Register, 72, 37346-37372.

van den Berg, M., Birnbaum, L., Bosveld, A. T. C., Brunström, B., Cook, P., Feeley, M., et al. (1998). Toxic equivalency factors (TEFs) for PCBs, PCDDs, PCDFs for humans and wildlife. Environmental Health Perspectives, 106, 775-792.

van den Berg, M., Birnbaum, L. S., Denison, M., De Vito, M., Farland, W., Feeley, M., et al. (2006). The 2005 World 
Health Organization re-evaluation of human and mammalian toxic equivalency factors for dioxins and dioxin-like compounds. Toxicological Science, 93, 223-241.

Walker, M. A., Cook, P. M., Butterworth, B. C., Zabel, E. W., \& Peterson, R. E. (1996). Potency of a complex mixture of polychlorinated dibenzo- $p$-dioxin, dibenzofuran, and biphenyl congeners compared 2,3,7,8-tetrachlorodibenzo- $p$-dioxin in causing fish early life stage mortality. Fundamental and Applied Toxicology, 30, 178-187.

Warner, K. A., Bonzongo, J. J., Roden, E. E., Ward, G. M., Green, A. C., Chaubey, I., et al. (2005). Effect of watershed parameters on mercury distribution in different environmental compartments in the Mobile Alabama River Basin, USA. Science of the Total Environment, 347, 187-207.

Whyte, J. J., Schmitt, C. J., \& Tillitt, D. E. (2004). The H4IIE cell bioassay as an indicator of dioxin-like chemicals in wildlife and the environment. Critical Reviews in Toxicology, 34, 1-83.

Wiemeyer, S. N., Lamont, T. G., Bunck, C. M., Sindelar, C. R., Gramlich, F. J., Fraser, J. D., et al. (1984). Organochlorine pesticide, polychlorobiphenyl, and mercury residues in bald eagle eggs-1969-79- and their relationships to shell thinning and reproduction. Archives of Environmental Contamination and Toxicology, 13, 529-549.

Wren, C. D., Hunter, D. B., Leatherland, J. F., \& Stokes, P. M. (1987). The effects of polychlorinated biphenyls and methylmercury, singly and in combination, on mink. I: Uptake and toxic responses. Archives of Environmental Contamination and Toxicology, 16, 441-447.

Yeardley Jr., R. B., Lazorchak, J. M., \& Paulsen, S. G. (1998). Elemental fish tissue contamination in northeastern U.S. lakes: evaluation of an approach to regional assessment. Environmental Toxicology and Chemistry, 17, 18751884.

Zappia, H. (2002). Organochlorine compounds and trace elements in fish tissue and streambed sediment in the mobile river basin, Alabama, Mississippi, and Georgia, 1998. Water-resources investigations Report 02-4160, US Geological Survey, Montgomery, Alabama. 